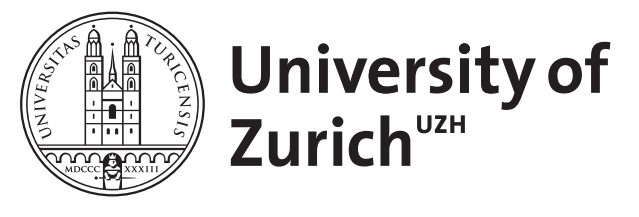

\title{
Optimal effort incentives in dynamic tournaments
}

Klein, Arnd Heinrich ; Schmutzler, Armin

\begin{abstract}
This paper analyzes two-stage rank-order tournaments. To influence efforts in the two periods, a principal can use the intertemporal prize structure and the weight of first-period performance in the second-period prize. These two instruments implement different sets of effort vectors. We characterize the optimal combination of prizes and weights as a function of parameters. For large parameter regions, the principal should only give a second-period prize, but use positive first-period performance weights. This holds no matter whether efforts in different periods are perfect or imperfect substitutes and whether the principal gives feedback on performance or not. We also generalize existing results on whether giving feedback is beneficial for the principal.
\end{abstract}

DOI: https://doi.org/10.1016/j.geb.2016.02.006

Posted at the Zurich Open Repository and Archive, University of Zurich

ZORA URL: https://doi.org/10.5167/uzh-147520

Journal Article

Accepted Version

Originally published at:

Klein, Arnd Heinrich; Schmutzler, Armin (2017). Optimal effort incentives in dynamic tournaments. Games and Economic Behavior, 103:199-224.

DOI: https://doi.org/10.1016/j.geb.2016.02.006 


\title{
Optimal Effort Incentives in Dynamic Tournaments
}

\author{
Arnd Heinrich Klein and Armin Schmutzler ${ }^{1}$
}

February 2016

\begin{abstract}
This paper analyzes two-stage rank-order tournaments. To influence efforts in the two periods, a principal can use the intertemporal prize structure and the weight of first-period performance in the secondperiod prize. These two instruments implement different sets of effort vectors. We characterize the optimal combination of prizes and weights as a function of parameters. For large parameter regions, the principal should only give a second-period prize, but use positive first-period performance weights. This holds no matter whether efforts in different periods are perfect or imperfect substitutes and whether the principal gives feedback on performance or not. We also generalize existing results on whether giving feedback is beneficial for the principal.
\end{abstract}

JEL: D02, D44

Keywords: dynamic tournaments, repeated contests, feedback, effort incentives

\footnotetext{
${ }^{1}$ Arnd Heinrich Klein: University of Zürich; arnd.klein@econ.uzh.ch. Armin Schmutzler: University of Zürich and CEPR; armin.schmutzler@econ.uzh.ch. We are grateful to Claudia Geiser, Andreas Hefti, Paul Heidhues, Stefan Jönsson, Igor Letina, John Morgan, Nick Netzer, Georg Nöldeke, Ron Siegel, two anonymous referees, and seminar audiences in Aarhus (Spring Meeting of Young Economists), Düsseldorf (Verein für Socialpolitik), Fresno (Conference on Tournaments, Contests and Relative Performance Evaluation), Lisbon (UECE), Lucerne (Zurich Workshop on Economics) and Zurich for helpful discussions. Shuo Liu provided excellent research assistance. Financial support of the Swiss National Science Foundation (grant numbers 131854 and 151688) is gratefully acknowledged.
} 


\section{Introduction}

Firms and other organizations often use dynamic tournaments to incentivize repeated effort provision. ${ }^{2}$ For instance, promotion tournaments and bonus systems are common. When designing such contests, a principal must ask how to induce the best possible intertemporal effort vector with a given budget. She can affect total effort and its distribution across periods though the prize structure, i.e., the division of a given prize sum between several periods. She can give one big prize after a long time or several small prizes in different periods. Apart from this prize policy, she has an alternative instrument for influencing the intertemporal effort distribution, namely the weight she gives to past performance when assigning prizes in later periods. We will show that, in spite of their superficial similarity, this weight policy and the prize policy have very different effects on effort streams, and we will identify the optimal combination of both instruments.

Specifically, we consider a two-period rank-order tournament with two riskneutral agents with identical and known abilities. ${ }^{3}$ The principal can split the prize money across two periods arbitrarily. If she awards a second-period prize, she also chooses a first-period performance weight. After the announcement of prize and weight policies, the agents choose effort levels in each period. The principal observes each agent's performance, a noisy measure of effort. In period 1, she awards the prize (if any) to the agent who performed better. In our benchmark case with feedback, she publicly announces the performance of both agents in the first period. In period 2, the agents choose efforts again. The principal then allocates the second-period prize to the agent for whom the weighted sum of first- and second-period performance is highest.

In line with the literature, we consider the case that a principal regards efforts in different periods and by different agents as perfect substitutes and thus maximizes total effort. Contrary to most of the literature, we also analyze the optimal policy for a principal who treats efforts in different periods as imperfect substitutes and wants to balance them across periods. This is important, because

\footnotetext{
${ }^{2} \mathrm{~A}$ well-known argument for tournaments is as follows. When performance is not verifiable, a principal who contracts directly on performance may claim that performance was low to save on performance pay. Tournaments reduce this incentive, because the total payments to the agents are independent of performance.

${ }^{3}$ To see the incentive effects of such tournaments most clearly, we abstract from the important issue of selecting the agent with the highest innate ability for a particular task.
} 
excessively low efforts in some period may cause large harm, which cannot even be compensated by an extremely large effort in other periods.

Our main contribution is that we compare the effects of prize and weight policies on effort streams. Moreover, we identify the optimal combination of both policies, depending on parameters. We use a simple example to show that a principal can induce the same maximal first-period effort and the same maximal second-period effort with each instrument. However, the two instruments implement different sets of effort vectors with positive efforts in both periods.

Next, we show that, under quite general conditions, the principal should give only a second-period prize, but with a positive first-period performance weight. The optimal first-period prize is positive only if the first-period performance measure is sufficiently precise. We then show that for quadratic cost functions and normally distributed observation errors, this condition never holds. Even with more general distributional assumptions, the optimal first-period prize is never higher than the second-period prize for imperfect substitutes and quadratic cost functions.

For the normal-quadratic example, we identify large gains from good design. The expected effort is at least $40 \%$ higher when a principal chooses prizes and weights optimally than when she carries out two identical independent tournaments with the same total prize sum.

Finally, we compare the results of the benchmark model with the no feedback case without communication of performance. ${ }^{4}$ We obtain similar results on the optimal combination of prizes and weights. Moreover, we generalize some existing results on whether a principle should give feedback.

The organization of the paper is as follows. Section 2 discusses related literature. In Section 3, we introduce the model. In Section 4, we analyze agent behavior. Section 5 uses a simple example to compare the incentive effects of pure prize policies and pure weight policies. Sections 6 and 7 characterize the optimal policy. Section 8 discusses the feedback policy. Section 9 concludes. The Appendix contains all non-trivial proofs.

\footnotetext{
${ }^{4}$ In the no revelation case, the game is static. The model thus becomes a special case of a multi-battle contest where agents compete simultaneously in a multiplicity of dimensions (see, e.g., Clark and Konrad 2007 and Kovenock and Roberson 2010).
} 


\section{Relation to the Literature}

Our paper focusses on the optimal choice of prizes and weights in dynamic tournaments. ${ }^{5}$ We are not aware of any work on the trade-off between these two instruments and their optimal combination. A small number of papers (Möller 2012, Clark et al. 2012, Clark and Nilsson 2013) derives the optimal prize structure in Tullock contests when good performers in period one have an exogenous advantage in period two, creating an asymmetry between the agents in the second period. ${ }^{6}$ However, these papers do not deal with the choice between prize and weight policies as alternatives for influencing the effort streams, which is central in our paper. Moreover, they neither analyze feedback policies nor do they allow for imperfect substitutes. ${ }^{7}$

Our results on optimal weights are closely related to the literature, but they provide additional insights. For instance, Meyer (1992) considers a setting similar to our case with feedback and a single prize, but with risk-averse agents. She shows that cost minimization requires a bias towards the first-period winner. ${ }^{8}$ Our analysis shows that the principal should also give a headstart when the first-period prize is higher than the second-period prize, when efforts are imperfect substitutes and when there is no feedback. Finally, we provide results on the determinants of the size of the bias. ${ }^{9}$

Several recent papers have dealt with feedback in dynamic tournaments (see Section 8). Aoyagi (2010) considers a two-period tournament similar to ours. However, he takes prizes and weights as exogenous: Unlike in our paper, there is

\footnotetext{
${ }^{5}$ Nitzan (1994) and Konrad (2009) provide surveys of the literature on tournaments. Another broadly related literature analyzes dynamic principal-agent relationships with moral hazard in a non-competitive setting. Lewis and Sappington (1997) examine how current incentives should optimally depend on past performance. Hansen (2013) and Chen and Chiu (2013) deal with the optimal revelation policy.

${ }^{6}$ These technological assumptions are also made by some authors who do not deal with the optimal prize structure (e.g., Schmitt et al. 2004, Grossmann and Dietl 2009, Grossmann 2011 and Baik and Lee 2000).

${ }^{7}$ Some papers derive the optimal distribution of prize money across stages in a two-period elimination tournament, where only the winners of the current period compete again in the next period. A seminal paper is Moldovanu and Sela (2006). Because elimination tournaments have a very different structure than our model, the results are difficult to compare to ours.

${ }^{8}$ See also Harbaugh and Ridlon (2011) and Ridlon and Shin (2013).

${ }^{9}$ Contrary to us, Meyer (1992) assumes that the size of the bias is fixed ex ante rather than a function of the performance difference in period 1.
} 
only one prize, and first and second-period performance receive the same weight. He shows that the expected effort is higher (lower) with feedback if marginal costs are concave (convex). ${ }^{10}$ We endogenize Aoyagi's assumptions on prizes and weights by analyzing under which circumstances the principal optimally chooses them in this way. Moreover, we show that the optimal feedback policy has the same features when these assumptions do not hold. Ederer (2010) introduces incomplete information about ability. The results are equivalent to those of Aoyagi (2010) if ability is non-complementary to effort. ${ }^{11}$ With complementarity, expected efforts may be higher with feedback than without, even for quadratic costs. ${ }^{12}$

Like us, Gershkov and Perry (2009) ask how a sequence of performance signals in a two-period setting should translate into prizes. The paper is hard to compare with ours, because the assumptions differ considerably. Most importantly, the authors assume that the principal can only use coarse information for interim performance evaluation (which, if any, agent was better).$^{13}$ Contrary to us, they do not ask whether and how the principal should spread a given prize sum over two periods. Instead, they investigate properties of the optimal mechanism when the principal can not only vary the prize distribution, but also the prize sum. Allowing such flexibility makes sense in their set-up (contrary to ours), because they assume performance to be verifiable, so that the principal is not tempted to report low performance (and thus pay low prizes). ${ }^{14}$

In brief, our paper contributes to the literature by showing how a principal

\footnotetext{
${ }^{10}$ Aoyagi (2010) allows for general objective functions of the principal and for partial feedback. Denter and Sisak (2013) show that effort may increase with feedback if marginal efforts are concave. They use their set-up to analyze the effect of polls on political campaign spending, allowing for an initial asymmetry before the beginning of the first period.

${ }^{11}$ Ederer and Fehr (2013) use a special case of this model with equal abilities.

${ }^{12}$ Other papers address feedback in dynamic tournaments under very different assumptions. Arbatskaya and Mialon (2012) analyze a lottery contest where first- and second-period efforts are complements. Goltsman and Mukherjee (2011) consider a contest in which the agents either succeed or fail, and the prize is given to the agent who succeeded more often. Zhang and Wang (2009) consider dynamic all-pay auctions with elimination.

${ }^{13}$ Also, Gershkov and Perry (2009) assume that the relation between winning probabilities and efforts is the same in both periods, while we allow for differences in the error structure. Finally, they only focus on maximization of total effort. Contrary to us, they allow for a technological relation between first-period effort and second-period performance.

${ }^{14}$ Like us, Gershkov and Perry (2009) have some results (e.g. Theorem 2) which are consistent with first-period efforts receiving positive weights. While they consider settings with and without midterm review, they do not directly analyze the ceteris paribus effect of giving feedback.
} 
should use prizes and weights to affect effort streams. Moreover, it provides robustness results regarding weight and feedback policies, and it justifies some assumptions previously used in the literature.

\section{The Model}

We consider a class of two-stage rank-order tournaments. Given a fixed budget $W>0$, a principal chooses an incentive system $\mathcal{I}=\left(\eta, W_{1}, \rho\right) \in \mathbb{R}^{+} \times[0, W] \times$ $\{0,1\}$ to be explained below. ${ }^{15}$ Given $\mathcal{I}$, agents $i \in\{1,2\}$ choose effort levels $e_{i t} \geq 0(t \in\{1,2\})$. The cost function $K_{i t}\left(e_{i t}\right)$ has the following properties:

Assumption 1: $K_{i t}$ is independent of $i$ and differentiable three times. It satisfies $K_{i t}^{\prime}>0, K_{i t}^{\prime \prime}>0, K_{i t}(0)=K_{i t}^{\prime}(0)=0 . K_{i t}^{\prime \prime \prime}\left(e_{i t}\right) \geq 0$ or $K_{i t}^{\prime \prime \prime}\left(e_{i t}\right) \leq 0$ must hold globally.

Thus, we can write $K_{t} \equiv K_{i t}$. Note that we allow first- and second-period cost functions to differ, reflecting potential differences in the two tasks.

The agents maximize expected utility and are risk-neutral. Utility is additively separable in period-specific income and costs. At the end of each period $t$, the principal observes performance, which is an imperfect effort measure $s_{i t}=e_{i t}+\varepsilon_{i t}$. The error term $\varepsilon_{i t}$ is independently distributed across agents and periods. In each period, the error distribution is the same for agent 1 as for agent 2. However, the error distribution in period 1 may differ from the one in period 2. This captures possible differences in the precision of monitoring for the two tasks. ${ }^{16}$

The principal awards the first-period prize $W_{1}$ to agent $i$ if $s_{i 1}>s_{j 1}$. Agent $i$ receives the second-period prize $W_{2}=W-W_{1}$ if $s_{i 2}+\eta s_{i 1}>s_{j 2}+\eta s_{j 1}$. The principal's choice of the first-period weight $\eta \in \mathbb{R}^{+}$thus determines the influence of past performance on the chance of winning in the second period.

Under a full feedback policy $(\rho=1)$, the principal makes the performance

\footnotetext{
${ }^{15}$ In Klein and Schmutzler (2014), we allow for $\eta<0$, but we show that this would never be optimal. Every $\eta<0$ is dominated by $|\eta|$, because $|\eta|$ will turn out to provide the same second-period incentives while strengthening first-period incentives.

${ }^{16}$ In a non-tournament setting, Ke et al. (2014) show that organizations optimally hire workers into easy-to-monitor jobs with low effort costs and then promote them into difficult-to-monitor jobs with high (marginal and absolute) effort costs. In our setting, this would correspond to $\sigma_{1}<\sigma_{2}$ and $K_{1}(e)<K_{2}(e), K_{1}^{\prime}(e)<K_{2}^{\prime}(e)$.
} 
of both agents public before they choose second-period efforts. ${ }^{17}$ Under a nofeedback policy $(\rho=0)$, the principal does not communicate the performance assessment. She does not even communicate who won the first-period prize, and she distributes both prizes at the end of period 2. In the following, we shall focus on the full feedback policy; except in Section 8.

The following notation is helpful to describe the solution of the game.

Definition 1 The error difference of agent $i$ in period $t(t=1,2)$ is $\Delta \varepsilon_{i t}=$ $\varepsilon_{i t}-\varepsilon_{j t}$. His relative first-period performance is $\Delta s_{i 1}=s_{i 1}-s_{j 1}=\Delta e_{i 1}+\Delta \varepsilon_{i t}$, where $\Delta e_{i t}=e_{i t}-e_{j t}$.

Clearly, $\Delta e_{i t}=-\Delta e_{j t}, \Delta \varepsilon_{i t}=-\Delta \varepsilon_{j t}, \Delta s_{i 1}=-\Delta s_{j 1}$. We make the following assumption on the error distributions:

Assumption $2 \Delta \varepsilon_{i t}$ is distributed as $F_{t}(s)$ on $\mathbb{R}$ with a symmetric, singlepeaked, strictly positive and continuously differentiable density $f_{t}(s)$.

This implies $f_{t}(s)=f_{t}(-s), f_{t}^{\prime}(s)=-f_{t}^{\prime}(-s)$ and $E\left(\Delta \varepsilon_{i t}\right)=0$.

For some results, we assume quadratic cost functions:

(C1) The cost function is $K_{t}\left(e_{i t}\right)=\frac{k_{t}}{2}\left(e_{i t}\right)^{2}$ with $k_{t}>0$.

We assume that, given a fixed prize budget, the principal's payoff is increasing in efforts. The efforts of different agents within periods are perfect substitutes for the principal. We allow first- and second period efforts to be perfect or imperfect substitutes. For perfect substitutes, the principal chooses the incentive system so as to maximize expected total efforts. For imperfect substitutes, she maximizes the expected product of first and second-period efforts. This corresponds to a complementarity that makes it desirable to have similar efforts in both periods.

\section{Agent behavior}

We focus on the full feedback policy, deferring the no feedback policy to Section 8. We first analyze the agents' equilibrium behavior for a given incentive system. The following simple result is stated without proof.

\footnotetext{
${ }^{17}$ In practice, the principal will typically not communicate a concrete number. Instead, she may communicate whatever relevant information she has to the agents, thereby creating a common understanding about their relative performance.
} 
Lemma 1 (i) The conditional probability that $s_{i 1}>s_{j 1}$ given $e_{i 1}$ and $e_{j 1}$ is $F_{1}\left(e_{i 1}-e_{j 1}\right)$.

(ii) The conditional probability that $s_{i 2}+\eta s_{i 1}>s_{j 2}+\eta s_{j 1}$ given $e_{i 2}, e_{j 2}$ and $\Delta s_{i 1}$ is $F_{2}\left(\eta \Delta s_{i 1}+e_{i 2}-e_{j 2}\right)$.

\subsection{General Analysis}

In period 2, a player's information set consists of all combinations of period 1 efforts and error differences that are consistent with the own first-period effort $e_{i 1}$ and the observed relative performance $\Delta s_{i 1}$. We use the Perfect Bayesian Equilibrium (PBE) to deal with this imperfect information (Mas-Colell et al. 1995, p. 285). ${ }^{18}$ A pure strategy $\sigma_{i}$ of player $i$ consists of a first-period choice $e_{i 1}$ and a function $E_{i 2}$ mapping information sets $\left(e_{i 1}, \Delta s_{i 1}\right)$ to actions $e_{i 2}$. If player $i$ chose $e_{i 1}$, observes $\Delta s_{i 1}$ and assumes that player $j$ plays the pure strategy $\sigma_{j}=$ $\left(e_{j 1}, E_{j 2}\right)$, he will assign probability one to the event that $\Delta \varepsilon_{i 1}=\Delta s_{i 1}-\Delta e_{i 1}$. We will assume that beliefs are formed in this way, without further mention.

\subsubsection{Second-period efforts}

Using Lemma 1(ii), the expected second-period payoff of agent $i$, conditional on relative first-period performance and second-period efforts, is

$$
U_{i 2}\left(e_{i 2}, e_{j 2}, \Delta s_{i 1}\right)=F_{2}\left(\eta \Delta s_{i 1}+\Delta e_{i 2}\right) W_{2}-K_{2}\left(e_{i 2}\right)
$$

Thus, the first period effort influences the second-period payoff via the firstperiod relative performance $\Delta s_{i 1}$. The first-order condition is

$$
f_{2}\left(\eta \Delta s_{i 1}+\Delta e_{i 2}\right) W_{2}=K_{2}^{\prime}\left(e_{i 2}\right)
$$

Though the game does not have any proper subgames because information sets in period 2 are not singletons, payoffs in period 2 are constant on information sets. We use this in the following definition.

Definition 2 The second-period effort game induced by $\Delta s_{i 1}$ is the game with

\footnotetext{
${ }^{18}$ The task is simplified because there are no off-equilibrium events to consider, as $f_{1}$ is strictly positive on $\mathbb{R}$. Moreover, period 1 enters player $i$ 's payoffs only via $\Delta s_{i 1}$ and $e_{i 1}$, so that the unobservable aspects of previous play (player $j$ 's effort choices) are irrelevant for the players' choices.
} 
players $i=1,2$, strategy spaces $X_{i}=\mathbb{R}^{+}$and payoffs given by (1) for $\left(e_{i 2}, e_{j 2}\right) \in$ $X_{i} \times X_{j}$.

We obtain the following result:

Lemma 2 Suppose $\rho=1$ (feedback) and $W_{2}>0$.

(i) In any equilibrium of the second-period effort game, efforts for $i=1,2$ are symmetric and satisfy

$$
e_{i 2}^{*}\left(\Delta s_{i 1}\right) \equiv e_{i 2}^{*}\left(\Delta s_{i 1} ; \eta, W_{2}, 1\right)=\left(K_{2}^{\prime}\right)^{-1}\left[f_{2}\left(\eta \Delta s_{i 1}\right) W_{2}\right]
$$

(ii) If costs are sufficiently convex, (3) defines the unique Nash equilibrium of the second-period effort game.

Lemma 2 has some simple comparative statics implications.

Corollary 1 Suppose $\rho=1, \eta>0$ and $W_{2}>0$. Then $e_{i 2}^{*}$ is decreasing in $\left|\Delta s_{i 1}\right|$ and $\eta$, and increasing in $W_{2}$ for $i=1,2$.

The result on $\left|\Delta s_{i 1}\right|$ states that a greater performance difference between the leader (the agent $i$ with $\Delta s_{i 1}>0$ ) and the laggard (the agent with $\Delta s_{i 1}<0$ ) reduces both players' effort in period $2 .{ }^{19}$ The other two results identify policy effects. In particular, increasing the first-period weight $\eta$ reduces second-period efforts.

In the PBE, the symmetric second-period equilibrium of the effort game is played after each realization of $\Delta s_{i 1}$. Thus, the expected second-period payoff, conditional on first-period performance, is

$$
U_{i 2}^{s}\left(\Delta s_{i 1}\right) \equiv U_{i 2}\left(e_{i 2}^{*}\left(\Delta s_{i 1}\right), e_{j 2}^{*}\left(-\Delta s_{i 1}\right), \Delta s_{i 1}\right)
$$

The expected second-period payoff, given first-period efforts, is

$$
U_{i 2}^{e}\left(e_{i 1}, e_{j 1}\right) \equiv E_{\Delta \varepsilon_{i 1}} U_{i 2}^{s}\left(\Delta e_{i 1}+\Delta \varepsilon_{i 1}\right) .
$$

\footnotetext{
${ }^{19}$ This result reflects the "well-known evaluation effect or lack-of-competition effect" (Ederer 2010, p. 742). It implies that the principal has an incentive to always report equal performances. This problem becomes negligible if the principal leaves the communication to disinterested parties from within or outside the organization.
} 


\subsubsection{First-period efforts}

Using Lemma 1(i), agent $i$ 's optimization problem in period 1 is

$$
\max _{e_{i 1} \geq 0} F_{1}\left(e_{i 1}-e_{j 1}\right) W_{1}+U_{i 2}^{e}\left(e_{i 1}, e_{j 1}\right)-K_{1}\left(e_{i 1}\right)
$$

The corresponding first-order conditions is

$$
f_{1}\left(\Delta e_{i 1}\right) W_{1}+\frac{\partial U_{i 2}^{e}}{\partial e_{i 1}}=K_{1}^{\prime}\left(e_{i 1}\right)
$$

The following definition is crucial for the intuition.

Definition 3 The intensity of second-period competition is given by

$$
C(\eta)=2 \int_{0}^{\infty} f_{2}(\eta s) f_{1}(s) d s .
$$

The logic of this definition is as follows. For each agent, $f_{1}(s)$ captures the density of the event that the relative first-period performance of this player is $s$ when efforts are symmetric (as in equilibrium). Since both players choose identical equilibrium efforts in the second period, $f_{2}(\eta s)=f_{2}(-\eta s)$ captures the density of the event that a strike of luck of one agent in period 2 exactly compensates a strike of luck of the other agent of size $s$ in period 1. Therefore, $C(\eta)$ measures the probability of the event that the second-period contest is a close run where a marginal effort increase of one agent will tip the balance of the secondperiod contest in his favor: When $C(\eta)$ is high, an agent who was lucky in the first period cannot be too sure about his winning prospects in the second period, and will therefore continue to put in some effort. $C(\eta)$ has simple properties. First,

$$
C^{\prime}(\eta)=2 \int_{0}^{\infty} s f_{2}^{\prime}(\eta s) f_{1}(s) d s<0 \text { for } \eta>0 .
$$

An increase in $\eta$ thus weakens second-period competition. Moreover,

$$
\text { (i) } C(\eta)>0 \text {; (ii) } C(0)=f_{2}(0) \text {; (iii) } C^{\prime}(0)=0
$$

We sometimes invoke a regularity condition to simplify the interpretation:

(C2) $\eta C(\eta)$ is increasing in $\eta$. 
This condition holds, for instance, in Example E1 below. The following result uses (6) to derive equilibrium efforts:

Proposition 1 Suppose $\rho=1$ (feedback).

(i) In any symmetric interior PBE, first-period efforts satisfy

$$
e_{1}^{*}\left(\eta, W_{1}, W_{2}, 1\right)=\left(K_{1}^{\prime}\right)^{-1}\left[f_{1}(0) W_{1}+\eta W_{2} C(\eta)\right] .
$$

(ii) Suppose the cost functions are sufficiently convex. Then (3) and (9) describe the unique symmetric PBE strategies.

We defer the discussion of second-order conditions to the appendix; there we will show that they require sufficiently convex cost functions.

By Proposition 1, if (C2) holds, a higher $\eta$ induces higher first-period effort. The term in brackets on the right-hand side of (9) is the marginal benefit from increasing $e_{i 1}$. The effect on the expected first-period payoff is $f_{1}(0) W_{1}$; the effect on the expected second-period payoff is $\eta W_{2} C(\eta)$, which is positive if $\eta>$ 0 . This term reflects the direct effect of higher first-period effort on second-period winning chances. The term does not capture strategic effects on the future efforts of the other player. Such effects are relevant in the game, but they cancel out in the symmetric equilibrium. ${ }^{20}$

We now characterize second-period efforts. Symmetry of the equilibrium in Proposition 1 implies $\Delta s_{i 1}=\Delta \varepsilon_{i 1}$. Using (3) and taking the expectation over $\Delta \varepsilon_{i 1}$, we obtain:

Corollary 2 The expected efforts in period 2 in the PBE of the feedback game described in Proposition 1 are

$$
E\left(e_{2}^{*}\left(\eta, W_{2}, 1\right)\right)=2 \int_{0}^{\infty}\left(K_{2}^{\prime}\right)^{-1}\left[f_{2}(\eta s) W_{2}\right] f_{1}(s) d s
$$

\footnotetext{
${ }^{20}$ If, for any first-period effort choice, a player knew he was ahead of the other player, he would have a strategic incentive to increase efforts to discourage player $j$ from exerting effort in the future, whereas the converse would hold for a player who knows he is behind the opponent. Since the game is stochastic, players have to consider the expected strategic effects, which can be positive or negative, but cancel out for identical first-period efforts.
} 


\subsection{A Normal-Quadratic Example}

To obtain sharper results, we introduce a simple example.

Example E1: The cost function is $K_{t}\left(e_{i t}\right)=\frac{k}{2} e_{i t}^{2}$ for $t=1,2$. The error difference $\Delta \varepsilon_{i t}$ is normally distributed with variance $\sigma_{t}^{2}{ }^{21}$

Example E1 satisfies Assumptions 1 and 2.

Corollary 3 In E1, a PBE exists. Equilibrium efforts with feedback are

$$
\begin{gathered}
e_{1}^{*}\left(\eta, W_{1}, W_{2}\right)=\frac{1}{k \sqrt{2 \pi}}\left(\frac{W_{1}}{\sigma_{1}}+\frac{\eta W_{2}}{\sqrt{\sigma_{2}^{2}+\sigma_{1}^{2} \eta^{2}}}\right) . \\
E\left(e_{2}^{*}\left(\eta, W_{2}, 1\right)\right)=\frac{W_{2}}{k \sqrt{2 \pi} \sqrt{\sigma_{2}^{2}+\sigma_{1}^{2} \eta^{2}}} .
\end{gathered}
$$

Lower marginal costs, higher second-period prize, lower first-period weight and higher first- and second-period precision induce higher second-period efforts. Analogous results hold for period one. First-period efforts also increase if the second-period precision increases: The parameter change makes first-period effort more worthwhile, because the positive effect on winning the second-period prize increases. Finally, a redistribution of the prize sum from period 2 to period 1 increases first-period efforts, because the positive effect of an increase in the firstperiod prize is always stronger than the negative effect of an identical decrease in the second-period prize.

\section{Prizes vs. Weights: An illustration}

We now use Example E1 to illustrate the fundamental difference between prize and weight policies that makes it worthwile to study the optimal combination of both instruments. We first ask which effort combinations can be implemented with a total budget $W$ with two independent tournaments $(\eta=0)$ with prizes $W_{1}$ and $W_{2}=W-W_{1}$. In this case, we speak of a pure prize policy. Second, we ask which effort combinations can be implemented with a pure weight policy, that is, with only one (second-period) prize $\left(W_{1}=0\right.$ and $\left.W_{2}=W\right)$, but arbitrary first-period weights $\eta$. We formulate the analysis for the feedback policy,

\footnotetext{
${ }^{21}$ A normally distributed error difference follows, for example, from normally distributed observation errors.
} 
but Corollary 6 below will show that an analogous argument works for the no feedback policy. ${ }^{22}$

For the purposes of this illustration, we assume that cost functions and performance measures are identical across periods. Our first result seems to confirm that there is no difference between prize and weight policies.

Observation 1: In Example E1 with $\sigma_{1}=\sigma_{2}=\sigma$, we obtain:

(i) A pure prize policy with $W_{1}=W$ and a pure weight policy with $\eta \rightarrow \infty$ both implement $\left(e_{1}, E\left(e_{2}\right)\right)=(W / k \sigma \sqrt{2 \pi}, 0)$.

(ii) A pure prize policy with $W_{1}=0$ and a pure weight policy with $\eta \rightarrow 0$ both implement $\left(e_{1}, E\left(e_{2}\right)\right)=(0, W / k \sigma \sqrt{2 \pi})$.

Thus, the maximal effort that the principal can induce with a pure prize policy in period 1 is the same as for a pure weight policy; similarly for period 2 . However, the two policies induce very different combinations of more balanced effort vectors with positive effort levels in both periods.

Observation 2: Independent of the prize distribution, the total expected effort for a pure prize policy is $W / k \sigma \sqrt{2 \pi}$. For a pure weight policy, the total expected effort is $W(1+\eta) / k \sigma \sqrt{2 \pi} \sqrt{\eta^{2}+1}$.

Figure 1 illustrates the observations. It depicts all effort vectors that can be implemented with each type of policy for $\sigma=1, k=1$ and $W=1$.

Reflecting Observation 1, both lines intersect the axes in the same point. The set of implementable effort vectors for a pure prize policy is a straight line with slope -1 , whereas for a pure weight policy it is a line that is concave to the origin. The figure also shows the principal's indifference curves with perfect and imperfect substitutes, respectively. The figure demonstrates that the advantages of weight policies for effort provision are particularly pronounced for balanced efforts $(\eta=1)$. These figures also imply that it is optimal for the principal to induce such balanced efforts in the symmetric example, even when efforts are perfect substitutes.

Intuitively, starting from an effort vector with all efforts concentrated in one period, the opportunity costs of inducing small positive effort levels in the other period are smaller with a weight policy than with a prize policy. Consider for

\footnotetext{
${ }^{22}$ Note that, since we focus on symmetric equilibria, and efforts within periods are perfect substitutes, we can write the principal's objective in terms of the efforts of only one agent here and in the following sections.
} 


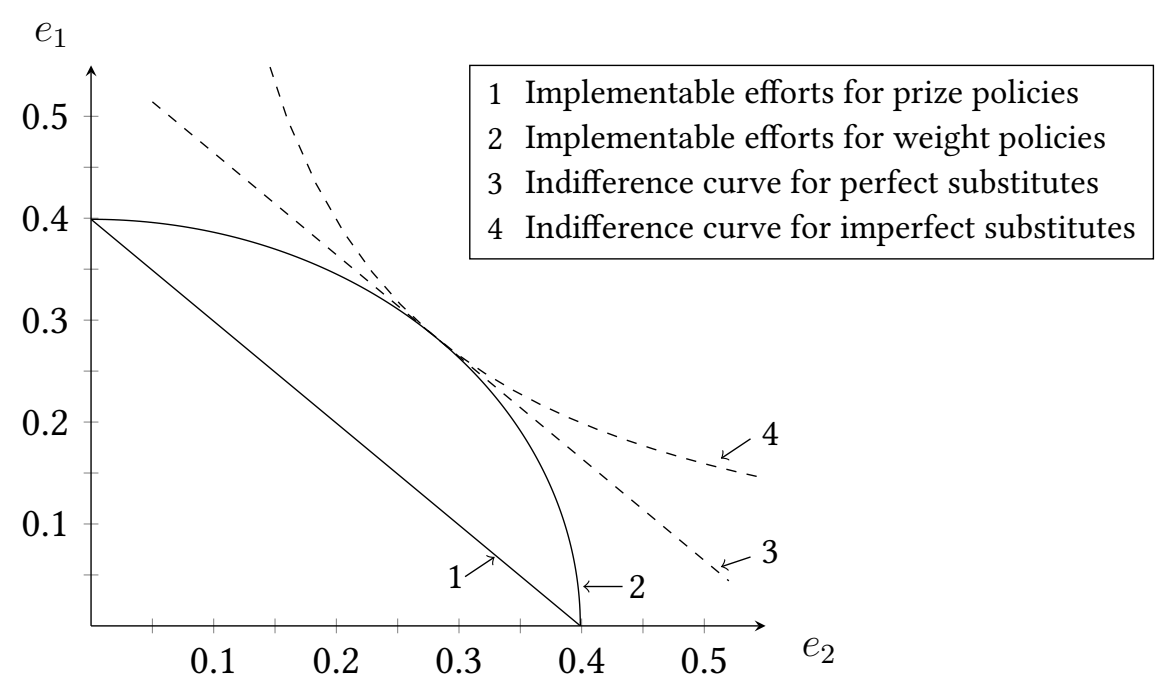

Figure 1: Implementable effort vectors for prize and weight policies

instance the first-order condition in period 2, which is $e_{2}=W_{2} C(0) / k=$ $W_{2} f_{2}(0) / k$ under a pure prize policy. Thus, inducing higher first-period efforts by marginally increasing $W_{1}$ has the opportunity cost that second-period efforts fall by $f_{2}(0) / k$, a positive constant that is independent of $W_{1}$. Under a pure weight policy, the first-order condition becomes $e_{2}=W C(\eta) / k$, so that the opportunity cost of marginally increasing $\eta$ is a reduction of the second-period effort by $W C^{\prime}(\eta) / k$, which depends directly on the resulting reduction in competition. As $C^{\prime}(0)$ is zero, the reduction in competition is negligible when one starts from a situation without first-period effort. The argument for the case without second-period effort is analogous.

In this special case, the principal prefers pure weight policies to pure prize policies. However, under more general conditions, positive first-period prizes will have a role to play, even though, at least for perfect substitutes, strong conditions are necessary for principals to benefit from offering two prizes. 


\section{Optimal Policy: Quadratic Cost Functions}

For the case of quadratic costs, we first characterize the optimal policy for perfect and imperfect intertemporal effort substitutes, respectively. ${ }^{23}$ Then we illustrate the size of the benefits resulting from an optimal design of the incentive system. We fix the total budget as $W$, so that $W_{2}=W-W_{1}$.

\subsection{Perfect Substitutes}

The principal's objective function for perfect substitutes is:

$$
V^{P}\left(\eta, W_{1}, \rho\right) \equiv e_{1}^{*}\left(\eta, W_{1}, W-W_{1}\right)+E\left(e_{2}^{*}\left(\eta, W-W_{1}, \rho\right)\right)
$$

The first main result characterizes the optimal policy. It is a special case of Lemma 5, which is stated for general cost functions (see Appendix 10.3 and the discussion in Section 7.1). ${ }^{24}$

Proposition 2 Suppose (C1) holds.

(i) If $\exists \eta \in \mathbb{R}^{+}$s.t. $f_{1}(0)<\left(k_{1} / k_{2}+\eta\right) C(\eta)$, then the optimal first-period prize is $W_{1}^{P}=0$. The optimal first period weight $\eta^{P}$ solves

$$
\left|\frac{C^{\prime}(\eta)}{C(\eta)}\right|=\frac{1}{k_{1} / k_{2}+\eta} .
$$

(ii) If $f_{1}(0) \geq\left(k_{1} / k_{2}+\eta\right) C(\eta) \forall \eta \in \mathbb{R}^{+}, W_{1}^{P}=W$.

The result implies that it is always optimal to give only one prize. To see this, note that, if (C1) holds, efforts in each period are linear functions of the prize in that period. As efforts are perfect substitutes, the principal focuses on the period for which the effect of the prize on the effort is higher.

When the principal gives a second-period prize, $\eta$ is determined by (14), which captures the trade-off between strengthening first-period incentives and

\footnotetext{
${ }^{23}$ In the following discussion, we assume that, for given error distributions and effort cost functions, second-order conditions hold for all allowable choices of the policy variables. This is for instance true for Example E1.

${ }^{24}$ We discuss Lemma 5 briefly below.
} 
weakening second-period competition. ${ }^{25}$ As the right-hand side of (14) is downwardsloping in $\eta$, the optimal $\eta$ falls as a result of any change in the error distribution that globally increases the sensitivity $\left|\frac{C^{\prime}(\eta)}{C(\eta)}\right|$ of second-period competition to $\eta$ : As $\left|\frac{C^{\prime}(\eta)}{C(\eta)}\right|$ shifts upward, the opportunity cost of using $\eta$ to increase first-period efforts (the reduction in future competition) increases. Thus, the principal should be more reluctant to use this instrument to induce first-period efforts. Figure 2 illustrates Proposition 2 for Example E1. It plots $\left|\frac{C^{\prime}(\eta)}{C(\eta)}\right|$ for three different values of the relative precision of the first- and second-period performance measures. We see that the optimal weight of first-period performance $\eta^{P}$ is higher if the second-period performance measure is imprecise compared to the first-period measure.

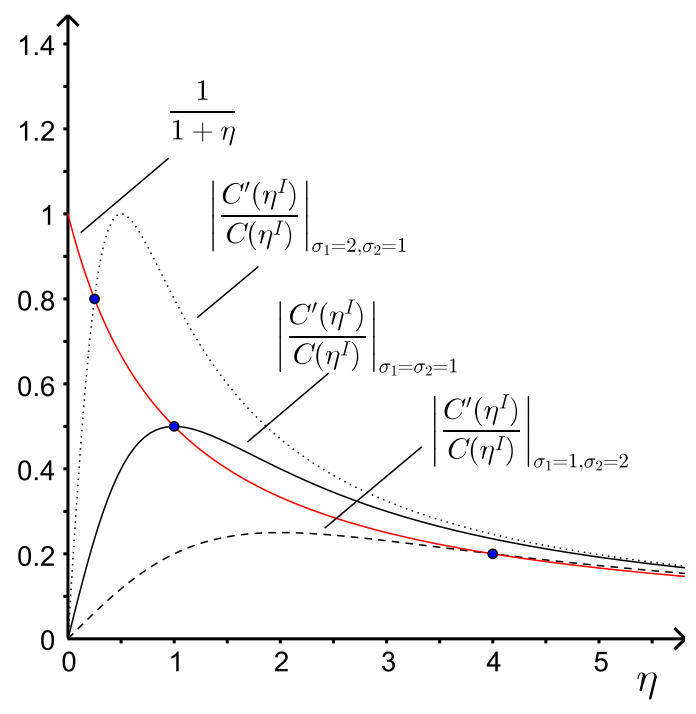

Figure 2: Necessary conditions for $\eta$ with perfect substitutes

Furthermore, the higher the ratio of first-period to second-period marginal costs, the lower is the optimal $\eta$. Note also that (14) and thus the optimal $\eta$ is independent of $W_{1} \cdot{ }^{26}$ Nevertheless, one should think of first-period prizes and weights as substitutes: As $W_{1}$ increases, $W_{2}=W-W_{1}$ falls. Thus, even if $\eta$ is unchanged, the marginal effect of higher first-period effort on the expected

\footnotetext{
${ }^{25}$ The proof shows that (14) not only holds for the optimal weight/prize combination, but for the optimal weight corresponding to any positive second-period prize.

${ }^{26}$ This is due to the fact that $W-W_{1}$ enters $\partial V^{P}\left(\eta, W_{1}\right) / \partial \eta$ multiplicatively (see (47)).
} 
future payoff $\left(\eta W_{2} C(\eta)\right)$ falls if $W_{1}$ increases, so that the principal relies less on the prospects of future prizes to induce first-period efforts.

Corollary 4 specifies the optimal policy for Example E1.

Corollary 4 In E1, $W_{1}^{P}=0$ and $\eta^{P}=\sigma_{2}^{2} / \sigma_{1}^{2}$.

Consistent with Proposition 2, it is optimal to give only a second-period prize with perfect substitutes. Incentives for first-period efforts come exclusively from $\eta$. The result endogenizes the assumption that $W_{1}=0$ and $\eta=1$ in Aoyagi (2010) for identically normally distributed error distributions: For $\sigma_{1}=\sigma_{2}$, this is the optimal combination of prizes and weights.

\subsection{Imperfect Substitutes}

The principal's objective function for imperfect substitutes is

$$
V^{I}\left(\eta, W_{1}, \rho\right) \equiv e_{1}^{*}\left(\eta, W_{1}, W-W_{1}\right) \cdot E\left(e_{2}^{*}\left(\eta, W-W_{1}, \rho\right)\right)
$$

Obviously, the principal wants to induce efforts in both periods. We can characterize the optimal policy for quadratic costs and, in particular, for the normalquadratic example.

Proposition 3 Suppose (C1) holds. The optimal $\left(W_{1}^{I}, \eta^{I}\right)$ satisfies one of the following properties:

$$
\begin{aligned}
& \text { (a) } W_{1}^{I}=0 \text { and }\left|\frac{C^{\prime}\left(\eta^{I}\right)}{C\left(\eta^{I}\right)}\right|=\frac{1}{2 \eta^{I}} \\
& \text { (b) } W_{1}^{I}=W \frac{f_{1}(0)-2 \eta^{I} C\left(\eta^{I}\right)}{2 f_{1}(0)-2 \eta^{I} C\left(\eta^{I}\right)} \in(0,1 / 2] \text { and }\left|\frac{C^{\prime}\left(\eta^{I}\right)}{C\left(\eta^{I}\right)}\right|=\frac{C\left(\eta^{I}\right)}{f_{1}(0)} \text {. }
\end{aligned}
$$

According to Proposition 3, there are two possibilities for $\left(W_{1}^{I}, \eta_{1}^{I}\right)$, both depicted in Figure 3.

According to (a), the first-period prize may be zero, in which case the optimal first-period weight satisfies a simple condition that depends exclusively on $C(\eta)$ (see point $A$ in Figure 3). As in the case of perfect substitutes, $\eta$ is lower the greater its adverse effect on future competition is. By (b), the first-period prize 


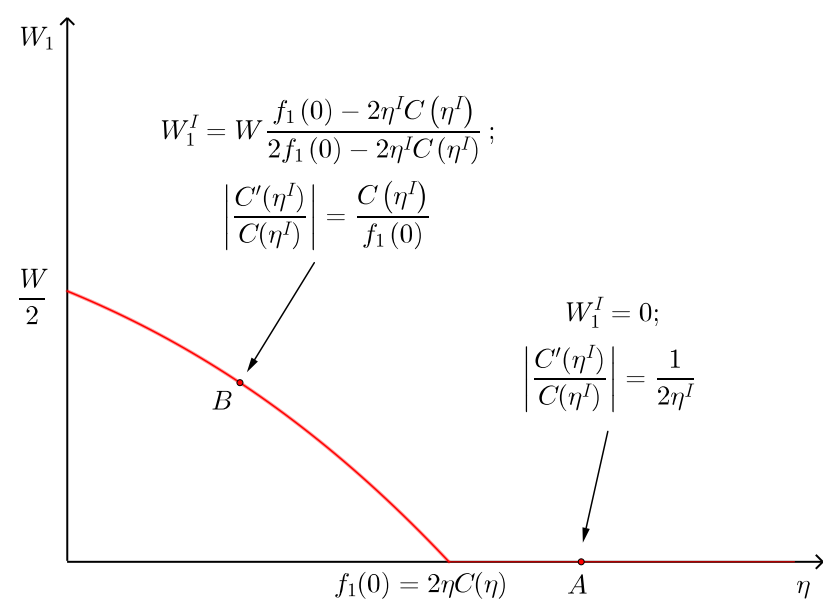

Figure 3: Necessary conditions for imperfect substitutes

may be positive, in which case the optimal first-period weight satisfies a condition that depends on error distributions not only via $C(\eta)$, but also via $f_{1}(0)$ directly, as captured by $W_{1}^{I}$ (see point $B$ in Figure 3 ). ${ }^{27}$ The error distributions determine which of the two cases in Proposition 3 applies. For instance, with normal error distributions, the first-period prize is zero (see Corollary 5 below).

Contrary to the case of perfect substitutes, Proposition 3 does not rule out positive prizes in both periods. Per-period efforts are linear in prizes, so that one of the prizes will typically have a more positive effect on total efforts. Nevertheless, the principal should not focus exclusively on this period, because she wants to balance efforts. On a related note, (b) states that the first-period prize is smaller than the second-period prize. This differs from perfect substitutes, for which it can be optimal to induce only first-period efforts. To obtain the desired balanced effort distribution, the principal should not give excessive first-period prizes, because she already provides incentives for first-period effort through $\eta$. In E1, we can say more about the optimum.

Corollary 5 In E1, necessary conditions for the optimum are $\eta^{I}=\sigma_{2} / \sigma_{1}$ and $W_{1}^{I}=0$.

The result resembles Corollary 4 for perfect substitutes, with variances replaced by standard deviations. Note that $\eta^{I}>\eta^{P}$ if and only if $\sigma_{2}<\sigma_{1}$ : Greater

\footnotetext{
${ }^{27}$ Note that $W_{1}^{I}(\eta)$ is typically not linear.
} 
precision of the second-period performance measure leads to higher secondperiod efforts than first-period efforts. Imperfect substitutes require balanced efforts, so that a greater weight of the first period is used to mitigate the asymmetry.

\subsection{The size of the benefits of optimal design}

We now use Example E1 to calculate the size of the benefits resulting from an optimal design of the incentive system with perfect substitutes. We compare the optimal choice of prizes and weights $\left(\left(\eta, W_{1}\right)=\left(\sigma_{2}^{2} / \sigma_{1}^{2}, 0\right)\right)$ with the case of two independent and identical tournaments $\left(\left(\eta, W_{1}\right)=(0, W / 2)\right)$. By optimally adjusting prizes and weights, the principal achieves a relative payoff increase of

$$
\Delta E^{P} \equiv \frac{V^{P}\left(\sigma_{2}^{2} / \sigma_{1}^{2}, 0\right)-V^{P}(0, W / 2)}{V^{P}(0, W / 2)}=\frac{2 \sqrt{\sigma_{1}^{2}+\sigma_{2}^{2}}-\left(\sigma_{1}+\sigma_{2}\right)}{\left(\sigma_{1}+\sigma_{2}\right)}
$$

Figure 4 shows how the total relative payoff increase from choosing the optimal incentive system depends on the standard deviations of the error distributions.

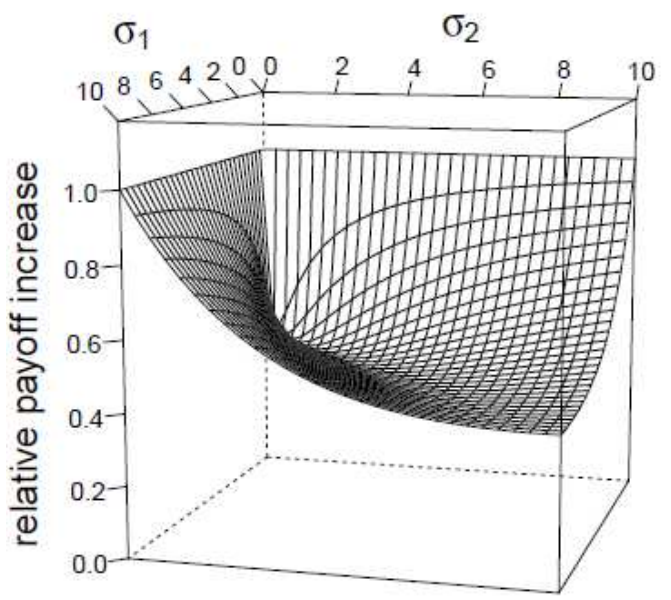

Figure 4: Relative payoff increase when setting $W_{1}$ and $\eta$ optimally

$\Delta E^{P}$ attains its minimum for $\sigma_{1}=\sigma_{2}$ at $\sqrt{2}-1 \approx 41 \%$. Thus, the percentage payoff increase from implementing the optimal policy is lowest if both performance measurements are equally precise. Figure 4 further shows that the more 
precise one of the performance measures is, the more the principal can benefit from implementing the optimal policy. ${ }^{28}$

\section{Optimal Policy: General cost functions}

With more general cost functions, we can still derive conditions for the optimal policy. While they are less straightforward to interpret, we can nevertheless obtain some insights.

\subsection{Prizes}

\subsubsection{Perfect Substitutes}

Proposition 2 on the optimal weights and prizes for quadratic cost functions and perfect substitutes follows from Lemma 5 in Appendix 10.3. This lemma, which assumes that $K_{t}^{\prime \prime \prime} \leq 0$, characterizes optimal prizes, conditional on weights. Specifically, it gives conditions under which the principal should only use a second period (first-period) prize. As in the case of quadratic cost functions, the principal should rely only on a second-period prize if the precision of the firstperiod signal is low. The assumption that $K_{t}^{\prime \prime \prime} \leq 0$ will turn out not to be a serious restriction: Corollary 6 in Section 8 shows that $K_{t}^{\prime \prime \prime} \leq 0$ corresponds to the case where feedback is optimal; if $K_{t}^{\prime \prime \prime}>0$, the principal should not give feedback.

\subsubsection{Imperfect Substitutes}

For imperfect substitutes, it is more difficult to characterize the optimal policy for general cost functions. One can show (for $K_{t}^{\prime \prime \prime} \leq 0$ ) that the optimal $W_{1}$ conditional on $\eta$ is zero whenever $f_{1}(0)<\eta C(\eta) .{ }^{29}$ Intutively, for any given first-period weight, positive first-period prizes should be avoided whenever the first-period signal is sufficiently noisy, but second-period competitiveness is sufficiently high that second-period prizes are useful.

\footnotetext{
${ }^{28}$ If one of the performance measures is very precise $\left(\sigma_{t} \approx 0\right)$, then $\Delta E^{P} \approx 1$.

${ }^{29}$ The result follows because $W_{1}=W$ cannot be optimal with imperfect substitutes, and (51) implies that the objective function is convex if $f_{1}(0)<\eta C(\eta)$; see Klein and Schmutzler (2014) for details.
} 


\subsection{Weights}

The following result generalizes an observation already made for quadratic cost functions:

Proposition 4 With feedback, the optimal weight is positive for perfect and imperfect substitutes for all positive second-period prizes $W_{2}$.

The result follows directly from Lemma 6 in the Appendix, according to which increasing $\eta$ marginally from zero increases first-period efforts, while there is no effect on second-period efforts. Proposition 4 states that performance evaluation should always have memory: Firms should consider not only the recent performance of employees, but also the performance in the distant past. As discussed for Example E1 in Section 5, this holds because, for $\eta=0$, the marginal effect of $\eta$ on first-period effort is positive and bounded away from zero (a first-order effect), whereas it is zero for second-period effort (a second-order effect). To understand the latter point, note that increasing $\eta$ has an adverse effect on secondperiod efforts because the second-period contest becomes more asymmetric, that is, less competitive $\left(C^{\prime}(\eta)<0\right)$. As $C^{\prime}(0)=0$, this adverse effect vanishes as $\eta$ approaches 0 .

As discussed in Section 2, several authors have argued that the principal should use positive first-period weights. The proof of Lemma 6 shows that this is even true for arbitrarily high first-period prizes. Moreover, it holds for imperfect as well as perfect substitutes.

\section{Feedback Policy}

We now suppose the principal does not give feedback about first-period performance. Section 8.1 characterizes the equilibrium. Section 8.2 compares the cases with and without feedback. Generalizing existing results, we show that the optimal feedback policy depends only on the sign of the third derivative of the cost function, with no difference for quadratic costs (C1). Hence, all results on the optimal prize/weight policy with (C1) translate directly to the case without feedback. In Section 8.3, we sketch some results for more general cost functions in the no feedback case. 


\subsection{No feedback}

\subsubsection{Equilibrium}

Under the no-feedback policy, agents simultaneously choose first- and secondperiod efforts according to

$$
\begin{gathered}
\max _{e_{i 1} \geq 0, e_{i 2} \geq 0} F_{1}\left(e_{i 1}-e_{j 1}\right) W_{1}+ \\
W_{2} \int_{-\infty}^{\infty} F_{2}\left(\eta\left(e_{i 1}-e_{j 1}+s\right)+e_{i 2}-e_{j 2}\right) f_{1}(s) d s-K_{1}\left(e_{i 1}\right)-K_{2}\left(e_{i 2}\right) .
\end{gathered}
$$

By Lemma 1(ii), the integral in (16) is the probability of winning the secondperiod prize, conditional on effort choices. We can use this to characterize the Nash equilibrium.

Proposition 5 Suppose $\rho=0$ (no feedback).

(i) In any symmetric interior Nash equilibrium, efforts must satisfy:

$$
\begin{aligned}
e_{1}^{*}\left(\eta, W_{1}, W_{2}, 0\right) & =\left(K_{1}^{\prime}\right)^{-1}\left[f_{1}(0) W_{1}+\eta W_{2} C(\eta)\right]>0 \\
e_{2}^{*}\left(\eta, W_{2}, 0\right) & =\left(K_{2}^{\prime}\right)^{-1}\left[W_{2} C(\eta)\right]>0 .
\end{aligned}
$$

(ii) If the cost functions are sufficiently convex, (17) and (18) describe the unique symmetric Nash equilibrium. ${ }^{30}$

Both effort levels reflect standard cost-benefit considerations. The marginal benefit of first-period efforts consists of the increased winning probability in period $2(\eta C(\eta))$ and period $1\left(f_{1}(0)\right)$.

\subsection{The effects of feedback}

By Propositions 1 and 5, first-period efforts in any symmetric equilibrium are non-stochastic and equal under both feedback policies; we thus write $e_{1}^{*}\left(\eta, W_{1}, W_{2}\right)$ for first-period equilibrium efforts. ${ }^{31}$ Using Jensen's inequality, we compare the

\footnotetext{
${ }^{30}$ In Appendix 10.5.2 we identify the meaning of "sufficient convexity". We also show that the second-order conditions hold locally for arbitrary convex cost function.

${ }^{31}$ The result reflects the fact that the marginal effect of first-period effort on the expected second-period payoff is identical under both policies. Intuitively, a marginal increase of $e_{i 1}$ has
} 
expected second-period efforts in the equilibria characterized by Propositions 1 and $5:^{32}$

Lemma $3 \forall \eta \in \mathbb{R}^{+}, W_{1}<W$ :

(i) If $K_{2}^{\prime \prime \prime} \geq 0$, then $e_{2}^{*}\left(\eta, W-W_{1}, 0\right) \geq E\left(e_{2}^{*}\left(\eta, W-W_{1}, 1\right)\right)$.

(ii) If $K_{2}^{\prime \prime \prime} \leq 0$, then $e_{2}^{*}\left(\eta, W-W_{1}, 0\right) \leq E\left(e_{2}^{*}\left(\eta, W-W_{1}, 1\right)\right)$.

For quadratic costs, (i) and (ii) imply that expected second-period efforts are equal under both feedback policies. Intuitively, $K_{2}^{\prime \prime \prime}$ matters because secondperiod efforts are the inverse of marginal costs for $\rho=0$ and the expectation of the inverse of marginal costs for $\rho=1$. Thus, concavity (convexity) of the inverse marginal costs is decisive for which regime yields higher efforts on expectation. ${ }^{33}$ A stark implication is that neither the other policy variables nor the exogenous parameters matter for the optimal feedback policy. Intuitively, this result should not hold when the third derivatives of cost functions switch sign; then the relation between second-period efforts with and without feedback will depend on details of the parameters and the policy. ${ }^{34}$ Moreover, even in the current set-up the remaining parameters matter for the extent to which efforts with and without feedback differ. ${ }^{35}$

A straightforward implication of Lemma 3 is that even if the principal has chosen the optimal parameters for a given feedback policy, switching to the

positive effects on the second-period payoff of player $i$ if it suffices to tip the balance in the contest in period 2 in his favor. The probability that this happens, which is captured by $C(\eta)$ for both players, is independent of whether information on $\Delta s_{i 1}$ is revealed to players before they choose second-period efforts. In this argument, it is important to start from the respective equilibrium, with equal efforts in both periods.

${ }^{32}$ Intuitively, with feedback, the agents base their second-period decisions on the revealed asymmetry between players, whereas, without feedback, the expected asymmetry is decisive. Compare second-period decisions with and without feedback for given effort choices in the first period: For error realizations where the asymmetry is low (high) relative to expectations, efforts will be higher (lower) with feedback than without.

${ }^{33}$ This result extends to non-separable cost functions if the third partial derivatives are positive (negative) for all first-period efforts: This follows from copying the analysis of the second-period game with the modified cost function,with $e_{1}$ playing a dummy role.

${ }^{34}$ For instance, if $K_{2}^{\prime \prime \prime}<0$ for small values of $e_{1}$ and $K_{2}^{\prime \prime \prime}>0$ for large values, one would expect the results for very low prizes to be as if $K_{2}^{\prime \prime \prime}<0$ and those for very large prizes to be as if $K_{2}^{\prime \prime \prime}$ $>0$.

${ }^{35}$ Trivially, for instance, when the second-period prize is small, the difference between the two policies becomes negligible, whereas it can be substantial for more general policies. 
other feedback policy is beneficial if the corresponding condition on $K_{2}^{\prime \prime \prime}$ holds. ${ }^{36}$ Hence, we have proven:

Corollary 6 The optimal feedback policy is the same for perfect and imperfect substitutes, with $\rho=0$ if $K_{2}^{\prime \prime \prime}>0$ and $\rho=1$ if $K_{2}^{\prime \prime \prime}<0$. For $K_{2}^{\prime \prime \prime}=0$, expected payoffs are independent of the feedback policy.

The result extends Aoyagi (2010) who shows that, for one prize $\left(W_{1}=0\right)$ and equal weights $(\eta=1)$ - the optimal parameters for Example E1 - the cost function completely determines the optimal feedback policy. ${ }^{37}$ Our result shows that this statement holds for arbitrary $W_{1}$ and $\eta$.

\subsection{Optimal Policy-Beyond Quadratic Costs}

By Lemma 3, feedback has no effect on (expected) efforts for quadratic costs. Thus, in this case, the results on prize and weight structure (Propositions 2 and 3 ) also hold without feedback. Lemma 8 in the Appendix characterizes the optimal prize structure without feedback for $K_{t}^{\prime \prime \prime} \geq 0$, where no feedback is superior by Lemma 3. The interpretation of the general results is similar as for quadratic costs: If the first-period contest is too noisy, it is optimal not to give a first-period prize. Moreover, arguments analogous to those in the proof of Proposition 4 show that, starting from $\eta=0$, a slight increase in $\eta$ has a positive first-order effect on $e_{1}$, but only a second-order effect on $e_{2}$. Hence, as in the case with feedback, it is optimal in the no feedback case to give a positive weight on past performance.

\section{Concluding Remarks}

This paper analyzes intertemporal effort provision in two-stage tournaments. A principal with a fixed budget faces two risk-neutral agents. She observes noisy effort signals in both periods. She aims at maximizing either total efforts (perfect

\footnotetext{
${ }^{36}$ To see this, note that Lemma 3 applies to all values of $\eta$ and $W_{1}$ and, in particular, to those that maximize $e_{2}^{*}\left(\eta, W-W_{1}, 0\right)$ or $E\left(e_{2}^{*}\left(\eta, W-W_{1}, 1\right)\right)$.

${ }^{37}$ Ederer (2010) also treats this case in his discussion of non-complementary abilities.
} 
substitutes) or the product of first- and second-period efforts (imperfect substitutes). She decides (i) how to spread prize money across the two periods, (ii) how to weigh performance in the two periods in the second period prize, and (iii) whether to reveal performance after the first period.

Prize and weight policies differ in their incentive effects, even though they seem similar at first sight. Our main results characterize the optimal combination of prizes and weights in terms of exogenous parameters, depending on whether efforts are perfect or imperfect substitutes. The analysis shows that, even when a principal can divide the prize arbitrarily, it is usually better to give only a secondperiod prize and rely on performance weights to incentivize first-period weights. We also show that the effects of different incentive structures can be quite substantial.

Several extensions are conceivable. First, one might ask, by going beyond the current model, what a rationale for using multiple prizes might be. Risk aversion is a natural candidate. Second, one might subject the hypotheses of our analysis to empirical tests. ${ }^{38}$

\section{References}

Aoyagi, M. (2010). Information feedback in a dynamic tournament. Games and Economic Behavior, 70(2):242-260.

Arbatskaya, M. and Mialon, H. M. (2012). Dynamic multi-activity contests. The Scandinavian fournal of Economics, 114(2):520-538.

Baik, K. H. and Lee, S. (2000). Two-stage rent-seeking contests with carryovers. Public Choice, 103(3-4):285-296.

Barut, Y. and Kovenock, D. (1998). The symmetric multiple prize all-pay auction with complete information. European fournal of Political Economy, 14(4):627-644.

Casas-Arce, P. and Martínez-Jerez, F. A. (2009). Relative performance compensation, contests, and dynamic incentives. Management Science, 55(8):1306-1320.

\footnotetext{
${ }^{38} \mathrm{Klein}$ (2015) confirms some of the comparative statics predictions in a laboratory experiment.
} 
Chen, B. R. and Chiu, Y. S. (2013). Interim performance evaluation in contract design. The Economic Fournal, 123(569):665-698.

Clark, D. J. and Konrad, K. A. (2007). Contests with multi-tasking. The Scandinavian fournal of Economics, 109(2):303-319.

Clark, D. J. and Nilssen, T. (2013). Learning by doing in contests. Public Choice, 156(1-2):329-343.

Clark, D. J., Nilssen, T., and Sand, J. Y. (2012). Motivating over time: Dynamic win effects in sequential contests. University of Oslo, Department of Economics Working Paper No. 28/2012.

Denter, P. and Sisak, D. (2013). Do polls create momentum in political competition? Tinbergen Institute Discussion Paper No. 13-169/VII.

Ederer, F. (2010). Feedback and motivation in dynamic tournaments. Fournal of Economics \& Management Strategy, 19(3):733-769.

Ederer, F. and Fehr, E. (2007). Deception and incentives: How dishonesty undermines effort provision. IZA Discussion Paper No. 3200.

Gershkov, A. and Perry, M. (2009). Tournaments with midterm reviews. Games and Economic Behavior, 66(1):162-190.

Goltsman, M. and Mukherjee, A. (2011). Interim performance feedback in multistage tournaments: The optimality of partial disclosure. Fournal of Labor Economics, 29(2):229-265.

Grossmann, M. (2011). Endogenous liquidity constraints in a dynamic contest. University of Zurich, Institute for Strategy and Business Economics Working Paper No. 148.

Grossmann, M. and Dietl, H. M. (2009). Investment behaviour in a twoperiod contest model. Fournal of Institutional and Theoretical Economics, 165(3):401-417.

Hansen, S. E. (2013). Performance feedback with career concerns. The fournal of Law, Economics, and Organization, 29(6):1279-1316. 
Harbaugh, R. and Ridlon, R. W. (2010). Handicapping under uncertainty in an allpay auction. Working paper. Retrieved 2015/01/15, from http://ssrn.com/abstract $=1688160$.

Hirata, D. (2014). A model of a two-stage all-pay auction. Mathematical Social Sciences, 68:5-13.

Ke, R., Li, J., and Powell, M. (2014). Managing careers in organisations. Unpublished working paper.

Klein, A. H. (2015). Optimal Incentives and Distributive Justice in Competitive Environments. $\mathrm{PhD}$ thesis, University of Zurich.

Klein, A. H. and Schmutzler, A. (2014). Optimal effort incentives in dynamic tournaments. CEPR Discussion Paper No. 10192.

Konrad, K. A. (2009). Strategy and Dynamics in Contests. New York: Oxford University Press.

Konrad, K. A. and Kovenock, D. (2009). Multi-battle contests. Games and Economic Behavior, 66(1):256-274.

Kovenock, D. and Roberson, B. (2010). Conflicts with multiple battlefields. CESifo Working Paper No. 3165.

Krumer, A. (2013). Best-of-two contests with psychological effects. Theory and Decision, 75(1):85-100.

Lewis, T. R. and Sappington, D. E. M. (1997). Penalizing success in dynamic incentive contracts: No good deed goes unpunished? The RAND fournal of Economics, 28(2):346-358.

Mas-Colell, A., Whinston, M. D., and Green, J. (1995). Microeconomic Theory. New York: Oxford University Press.

Meyer, M. A. (1992). Biased contests and moral hazard: Implications for career profiles. Annales d'Économie et de Statistique, 25/26:165-187.

Moldovanu, B. and Sela, A. (2001). The optimal allocation of prizes in contests. The American Economic Review, 91(3):542-558. 
Moldovanu, B. and Sela, A. (2006). Contest architecture. Fournal of Economic Theory, 126(1):70-96.

Möller, M. (2012). Incentives versus competitive balance. Economics Letters, 117(2):505-508.

Nitzan, S. (1994). Modelling rent-seeking contests. European fournal of Political Economy, 10:41-60.

Ridlon, R. and Shin, J. (2013). Favoring the winner or loser in repeated contests. Marketing Science, 32(5):768-785.

Schmitt, P., Shupp, R., Swope, K., and Cadigan, J. (2004). Multi-period rentseeking contests with carryover: Theory and experimental evidence. Economics of Governance, 5(3):187-211.

Schweinzer, P. and Segev, E. (2012). The optimal prize structure of symmetric tullock contests. Public Choice, 153(1-2):69-82.

Sela, A. (2011). Best-of-three all-pay auctions. Economics Letters, 112(1):67-70.

Yildirim, H. (2005). Contests with multiple rounds. Games and Economic Behavior, 51(1):213-227.

Zhang, J. and Wang, R. (2009). The role of information revelation in elimination contests. The Economic fournal, 119(536):613-641. 


\section{Appendix}

\subsection{Behavior of the Agents ${ }^{39}$}

\subsubsection{Proof of Lemma 2}

(i) Equilibrium efforts must be positive because $f_{2}>0$ by Assumption 2 and $K_{2}^{\prime}(0)=0$ by Assumption 1. Since $f_{2}$ is symmetric by Assumption 2 and

$$
-\left(\eta \Delta s_{11}+\Delta e_{12}\right)=\eta \Delta s_{21}+\Delta e_{22}
$$

the left-hand side of the first-order condition (2) is equal for both agents. Hence, the second-period efforts are the same for both agents. Thus, (2) becomes $f_{2}\left(\eta \Delta s_{i 1}\right) W_{2}=$ $K_{2}^{\prime}\left(e_{i 2}\right)$. As $K_{2}^{\prime \prime}>0$ by Assumption $1, K_{2}^{\prime}$ is strictly increasing and thus invertible. Thus (3) must hold in any equilibrium.

(ii) The following inequality guarantees that the second-period payoffs (1) of player $i$ are strictly concave in $e_{i 2}$ :

$$
f_{2}^{\prime}\left(\eta \Delta s_{i 1}+\Delta e_{i 2}\right) W_{2}<K_{2}^{\prime \prime}\left(e_{i 2}\right) \forall \Delta s_{i 1} \in \mathbb{R}, e_{i 2}, e_{j 2} \in \mathbb{R}^{+} .
$$

(19) requires $K_{2}$ to be sufficiently convex. ${ }^{40}$ If it holds globally, the first-order conditions (2) characterize a Nash equilibrium. Moreover, the equilibrium is unique, as (3) must hold in any equilibrium by Part (i) of the lemma.

\subsubsection{Proof of Corollary 1}

The inverse function theorem yields

$$
\left[\left(K_{2}^{\prime}\right)^{-1}\right]^{\prime}\left(f_{2}\left(\eta \Delta s_{i 1}\right) W_{2}\right)=\frac{1}{K_{2}^{\prime \prime}\left(\left(K_{2}^{\prime}\right)^{-1}\left(f_{2}\left(\eta \Delta s_{i 1}\right) W_{2}\right)\right)} .
$$

\footnotetext{
${ }^{39}$ The proofs in this section generalize Aoyagi (2010) and Ederer (2010) (for noncomplementary abilities) who assume $W_{1}=0$ and $\eta=1$.

${ }^{40}$ By Assumption 2, $f_{2}^{\prime}\left(\eta \Delta s_{i 1}+\Delta e_{i 2}\right)<0$ if $\eta \Delta s_{i 1}+\Delta e_{i 2}>0$, so that (19) always holds in this case. For the case that $\eta \Delta s_{i 1}+\Delta e_{i 2}<0$, suppose $f_{2}^{\prime}$ is bounded above. Then (19) holds globally if $K_{2}^{\prime \prime}$ has a sufficiently high lower bound.
} 
Thus (3) implies

$$
\begin{aligned}
\frac{\partial e_{i 2}}{\partial \Delta s_{i 1}} & =\frac{\eta f_{2}^{\prime}\left(\eta \Delta s_{i 1}\right) W_{2}}{K_{2}^{\prime \prime}\left(\left(K_{2}^{\prime}\right)^{-1}\left(f_{2}\left(\eta \Delta s_{i 1}\right) W_{2}\right)\right)} \\
\frac{\partial e_{i 2}}{\partial \eta} & =\frac{\Delta s_{i 1} f_{2}^{\prime}\left(\eta \Delta s_{i 1}\right) W_{2}}{K_{2}^{\prime \prime}\left(\left(K_{2}^{\prime}\right)^{-1}\left(f_{2}\left(\eta \Delta s_{i 1}\right) W_{2}\right)\right)} ; \\
\frac{\partial e_{i 2}}{\partial W_{2}} & =\frac{f_{2}\left(\eta \Delta s_{i 1}\right)}{K_{2}^{\prime \prime}\left(\left(K_{2}^{\prime}\right)^{-1}\left(f_{2}\left(\eta \Delta s_{i 1}\right) W_{2}\right)\right)} .
\end{aligned}
$$

By Assumption 1, $K_{2}^{\prime \prime}>0$. By Assumption 2, if $\Delta s_{i 1}<(>) 0 \wedge \eta>0 \wedge W_{2}>0$, then $\eta f_{2}^{\prime}\left(\eta \Delta s_{i 1}\right)<0$ and thus $\frac{\partial e_{i 2}}{\partial \Delta s_{i 1}}>(<) 0$. This implies that $e_{i 2}$ is decreasing in $\left|\Delta s_{i 1}\right|$. Similar arguments show that $\frac{\partial e_{i 2}}{\partial \eta}<0$. Since $f_{2}>0$ by Assumption 2, we have $\frac{\partial e_{i 2}}{\partial W_{2}}>0$.

\subsubsection{Proof of Proposition 1}

(i) We first derive expressions for $\frac{\partial U_{i 2}^{e}}{\partial e_{i 1}}$ for symmetric first-period efforts. This allows us to state the FOC.

\section{Lemma 4}

$$
\left.\frac{\partial U_{i 2}^{e}}{\partial e_{i 1}}\right|_{e_{i 1}=e_{j 1}}=\eta W_{2} C(\eta)
$$

Proof. Applying the envelope theorem to (4), we obtain

$$
\frac{d U_{i 2}^{s}\left(\Delta s_{i 1}\right)}{d \Delta s_{i 1}}=\frac{\partial U_{i 2}}{\partial e_{j 2}} \frac{\partial e_{j 2}^{*}\left(-\Delta s_{i 1}\right)}{\partial \Delta s_{i 1}}+\frac{\partial U_{i 2}}{\partial \Delta s_{i 1}} .
$$

Using (20) and the symmetry of the density (Assumption 2),

$$
\frac{\partial e_{j 2}^{*}\left(-\Delta s_{i 1}\right)}{\partial \Delta s_{i 1}}=\frac{\partial e_{i 2}\left(\Delta s_{i 1}\right)}{\partial \Delta s_{i 1}}=\frac{\eta f_{2}^{\prime}\left(\eta \Delta s_{i 1}\right) W_{2}}{K_{2}^{\prime \prime}\left(\left(K_{2}^{\prime}\right)^{-1}\left(f_{2}\left(\eta \Delta s_{i 1}\right) W_{2}\right)\right)} .
$$

(1) implies

$$
\begin{gathered}
\frac{\partial U_{i 2}}{\partial e_{j 2}}=-f_{2}\left(\eta \Delta s_{i 1}+\Delta e_{i 2}\right) W_{2}, \\
\frac{\partial U_{i 2}}{\partial \Delta s_{i 1}}=\eta f_{2}\left(\eta \Delta s_{i 1}+\Delta e_{i 2}\right) W_{2} .
\end{gathered}
$$


Using these equations in (24) and inserting $\Delta e_{i 2}=0$, we obtain

$$
\frac{d U_{i 2}^{s}}{d \Delta s_{i 1}}=-\frac{\eta f_{2}\left(\eta \Delta s_{i 1}\right) f_{2}^{\prime}\left(\eta \Delta s_{i 1}\right) W_{2}^{2}}{K_{2}^{\prime \prime}\left(\left(K_{2}^{\prime}\right)^{-1}\left(f_{2}\left(\eta \Delta s_{i 1}\right) W_{2}\right)\right)}+\eta f_{2}\left(\eta \Delta s_{i 1}\right) W_{2} .
$$

Using this in (5), we obtain

$$
\begin{gathered}
\frac{\partial U_{i 2}^{e}}{\partial e_{i 1}}= \\
\int_{-\infty}^{\infty}\left[-\frac{\eta f_{2}\left(\eta\left(\Delta e_{i 1}+s\right)\right) f_{2}^{\prime}\left(\eta\left(\Delta e_{i 1}+s\right)\right) W_{2}^{2}}{K_{2}^{\prime \prime}\left(\left(K_{2}^{\prime}\right)^{-1}\left(f_{2}\left(\eta\left(\Delta e_{i 1}+s\right)\right) W_{2}\right)\right)}+\eta f_{2}\left(\eta\left(\Delta e_{i 1}+s\right)\right) W_{2}\right] f_{1}(s) d s \\
=\eta W_{2} \int_{-\infty}^{\infty} f_{2}\left(\eta\left(\Delta e_{i 1}+s\right)\right) f_{1}(s) d s- \\
\eta W_{2}^{2} \int_{-\infty}^{\infty} \frac{f_{2}\left(\eta\left(\Delta e_{i 1}+s\right)\right) f_{2}^{\prime}\left(\eta\left(\Delta e_{i 1}+s\right)\right)}{K_{2}^{\prime \prime}\left(\left(K_{2}^{\prime}\right)^{-1}\left(f_{2}\left(\eta\left(\Delta e_{i 1}+s\right)\right) W_{2}\right)\right)} f_{1}(s) d s
\end{gathered}
$$

Let

$$
\begin{aligned}
& A:=\int_{-\infty}^{\infty} f_{2}\left(\eta\left(\Delta e_{i 1}+s\right)\right) f_{1}(s) d s \\
& B:=\int_{-\infty}^{\infty} \frac{f_{2}\left(\eta\left(\Delta e_{i 1}+s\right)\right) f_{2}^{\prime}\left(\eta\left(\Delta e_{i 1}+s\right)\right)}{K_{2}^{\prime \prime}\left(\left(K_{2}^{\prime}\right)^{-1}\left(f_{2}\left(\eta\left(\Delta e_{i 1}+s\right)\right) W_{2}\right)\right)} f_{1}(s) d s
\end{aligned}
$$

With this notation,

$$
\frac{\partial U_{i 2}^{e}}{\partial e_{i 1}}=\eta W_{2} A-\eta W_{2}^{2} B .
$$

Substituting $s=t-\Delta e_{i 1}$ and $d s=d t$ in $A$ and decomposing the integral gives

$$
A=\int_{-\infty}^{0} f_{2}(\eta t) f_{1}\left(t-\Delta e_{i 1}\right) d t+\int_{0}^{\infty} f_{2}(\eta t) f_{1}\left(t-\Delta e_{i 1}\right) d t
$$

Let $u=-t$. Symmetry of $f_{1}$ and $f_{2}$ by Assumption 2 implies $f_{2}(\eta t)=f_{2}(\eta u)$ and $f_{1}\left(t-\Delta e_{i 1}\right)=f_{1}\left(u+\Delta e_{i 1}\right)$. Hence,

$$
\int_{-\infty}^{0} f_{2}(\eta t) f_{1}\left(t-\Delta e_{i 1}\right) d t=\int_{0}^{\infty} f_{2}(\eta u) f_{1}\left(u+\Delta e_{i 1}\right) d u
$$


Thus,

$$
\begin{aligned}
A & =\int_{0}^{\infty} f_{2}(\eta u) f_{1}\left(u+\Delta e_{i 1}\right) d u+\int_{0}^{\infty} f_{2}(\eta t) f_{1}\left(t-\Delta e_{i 1}\right) d t \\
& =\int_{0}^{\infty} f_{2}(\eta t)\left[f_{1}\left(t+\Delta e_{i 1}\right)+f_{1}\left(t-\Delta e_{i 1}\right)\right] d t .
\end{aligned}
$$

Substituting $s=t-\Delta e_{i 1}$ and $d s=d t$ in $B$ and decomposing the integral, we obtain

$$
B=\int_{-\infty}^{0} \frac{f_{2}(\eta t) f_{2}^{\prime}(\eta t) f_{1}\left(t-\Delta e_{i 1}\right)}{K_{2}^{\prime \prime}\left(\left(K_{2}^{\prime}\right)^{-1}\left(f_{2}(\eta t) W_{2}\right)\right)} d t+\int_{0}^{\infty} \frac{f_{2}(\eta t) f_{2}^{\prime}(\eta t) f_{1}\left(t-\Delta e_{i 1}\right)}{K_{2}^{\prime \prime}\left(\left(K_{2}^{\prime}\right)^{-1}\left(f_{2}(\eta t) W_{2}\right)\right)} d t
$$

Again using $u=-t$ and appealing to symmetry, $f_{2}(\eta t)=f_{2}(\eta u), f_{2}^{\prime}(\eta t)=-f_{2}^{\prime}(\eta u)$ and $f_{1}\left(t-\Delta e_{i 1}\right)=f_{1}\left(u+\Delta e_{i 1}\right)$. Thus

$$
\int_{-\infty}^{0} \frac{f_{2}(\eta t) f_{2}^{\prime}(\eta t) f_{1}\left(t-\Delta e_{i 1}\right)}{K_{2}^{\prime \prime}\left(\left(K_{2}^{\prime}\right)^{-1}\left(f_{2}(\eta t) W_{2}\right)\right)} d t=\int_{0}^{\infty} \frac{f_{2}(\eta u)\left(-f_{2}^{\prime}(\eta u)\right) f_{1}\left(u+\Delta e_{i 1}\right)}{K_{2}^{\prime \prime}\left(\left(K_{2}^{\prime}\right)^{-1}\left(f_{2}(\eta u) W_{2}\right)\right)} d u
$$

Hence,

$$
\begin{aligned}
B & =\int_{0}^{\infty} \frac{-f_{2}(\eta u) f_{2}^{\prime}(\eta u) f_{1}\left(u+\Delta e_{i 1}\right)}{K_{2}^{\prime \prime}\left(\left(K_{2}^{\prime}\right)^{-1}\left(f_{2}(\eta u) W_{2}\right)\right)} d u+\int_{0}^{\infty} \frac{f_{2}(\eta t) f_{2}^{\prime}(\eta t) f_{1}\left(t-\Delta e_{i 1}\right)}{K_{2}^{\prime \prime}\left(\left(K_{2}^{\prime}\right)^{-1}\left(f_{2}(\eta t) W_{2}\right)\right)} d t \\
& =\int_{0}^{\infty} \frac{f_{2}(\eta t) f_{2}^{\prime}(\eta t)\left[-f_{1}\left(t+\Delta e_{i 1}\right)+f_{1}\left(t-\Delta e_{i 1}\right)\right]}{K_{2}^{\prime \prime}\left(\left(K_{2}^{\prime}\right)^{-1}\left(f_{2}(\eta t) W_{2}\right)\right)} d t .
\end{aligned}
$$

Substituting the expressions for $A$ and $B$ into (25) and using $s=t$, we obtain

$$
\begin{gathered}
\frac{\partial U_{i 2}^{e}}{\partial e_{i 1}}=\eta W_{2} \int_{0}^{\infty} f_{2}(\eta s)\left[f_{1}\left(s+\Delta e_{i 1}\right)+f_{1}\left(s-\Delta e_{i 1}\right)\right] d s \\
+\eta W_{2}^{2} \int_{0}^{\infty} \frac{f_{2}(\eta s) f_{2}^{\prime}(\eta s)\left[f_{1}\left(s+\Delta e_{i 1}\right)-f_{1}\left(s-\Delta e_{i 1}\right)\right]}{K_{2}^{\prime \prime}\left(\left(K_{2}^{\prime}\right)^{-1}\left(f_{2}(\eta s) W_{2}\right)\right)} d s .
\end{gathered}
$$

With $\Delta e_{i 1}=0$, we obtain (23).

Together, (6) and Lemma 4 imply

$$
f_{1}(0) W_{1}+\eta W_{2} C(\eta)=K_{1}^{\prime}\left(e_{i 1}\right)
$$

By Assumption 1, $K_{1}^{\prime}$ is invertible. We thus obtain (9) as a necessary condition for any symmetric interior PBE. 
(ii) By Lemma 2(ii) (2) implies sequential rationality in the second period. From the discussion at the beginning of Section 4.1, beliefs are consistent.

As $K_{1}^{\prime}(0)=0$ by Assumption 1, efforts must be positive in any symmetric equilibrium. Thus, by Part (i), (9) is a necessary condition for an equilibrium. The second-order condition for player $i$ is

$$
f_{1}^{\prime}\left(\Delta e_{i 1}\right) W_{1}+\frac{\partial^{2} U_{i 2}^{e}}{\partial e_{i 1}^{2}}<K_{1}^{\prime \prime}\left(e_{i 1}\right) \forall e_{i 1}, e_{j 1} \in \mathbb{R}^{+} .
$$

Inserting (26) in (27) gives

$$
\begin{aligned}
& f_{1}^{\prime}\left(\Delta e_{i 1}\right) W_{1}+\eta W_{2} \int_{0}^{\infty} f_{2}(\eta s)\left[f_{1}^{\prime}\left(s+\Delta e_{i 1}\right)-f_{1}^{\prime}\left(s-\Delta e_{i 1}\right)\right] d s+ \\
& \eta W_{2}^{2} \int_{0}^{\infty} \frac{f_{2}(\eta s) f_{2}^{\prime}(\eta s)\left[f_{1}^{\prime}\left(s+\Delta e_{i 1}\right)+f_{1}^{\prime}\left(s-\Delta e_{i 1}\right)\right]}{K_{2}^{\prime \prime}\left(\left(K_{2}^{\prime}\right)^{-1}\left(f_{2}(\eta s) W_{2}\right)\right)} d s<K_{1}^{\prime \prime}\left(e_{i 1}\right) .
\end{aligned}
$$

The left-hand side of this inequality is decreasing in $K_{2}^{\prime \prime}$, while the right-hand side is increasing in $K_{1}^{\prime \prime}$. For given policy parameters and distributions such that the slopes of $f_{1}$ and $f_{2}$ are bounded, (28) therefore holds as long as $K_{1}^{\prime \prime}$ and $K_{2}^{\prime \prime}$ are sufficently large. If these conditions hold globally, (9) thus describes an equilibrium, which is the unique symmetric equilibrium.

\subsubsection{Proof of Corollary 2}

Symmetry of the equilibrium implies $\Delta s_{i 1}=\Delta \varepsilon_{i 1}$. Hence, (3) implies

$$
e_{i 2}^{*}\left(\Delta s_{i 1}, \eta, W_{2}, 1\right)=\left(K_{2}^{\prime}\right)^{-1}\left(f_{2}\left(\eta \Delta \varepsilon_{i 1}\right) W_{2}\right) .
$$

Taking the expectation over $\Delta \varepsilon_{i 1}$, we obtain

$$
E_{\Delta \varepsilon_{i 1}}\left(e_{i 2}^{*}\left(\Delta s_{i 1}, \eta, W_{2}, 1\right)\right)=\int_{-\infty}^{\infty}\left(K_{2}^{\prime}\right)^{-1}\left(f_{2}(\eta s) W_{2}\right) f_{1}(s) d s .
$$

From the symmetry of the density by Assumption 2, we get (10).

\subsubsection{Proof of Corollary 3}

\section{Part 1: Auxilliary Results}


We will first provide several auxiliary results. Note that $2 \int_{0}^{x} \exp \left(-t^{2}\right) d t / \sqrt{\pi}$ is the error function, for which

$$
2 \int_{0}^{\infty} \exp \left(-t^{2}\right) d t / \sqrt{\pi}=1
$$

Next, for E1,

$$
f_{t}(s)=\frac{1}{\sigma_{t} \sqrt{2 \pi}} \exp \left(-\frac{s^{2}}{2 \sigma_{t}^{2}}\right)
$$

Hence,

$$
\begin{aligned}
f_{t}^{\prime}(s) & =-\frac{1}{\sqrt{2 \pi}} \frac{s}{\sigma_{t}^{3}} \exp \left(-\frac{s^{2}}{2 \sigma_{t}^{2}}\right) ; \\
f_{t}^{\prime \prime}(s) & =\frac{1}{\sqrt{2 \pi}} \frac{s^{2}-\sigma_{t}^{2}}{\sigma_{t}^{5}} \exp \left(-\frac{s^{2}}{2 \sigma_{t}^{2}}\right) ; \\
f_{t}^{\prime \prime \prime}(s) & =\frac{1}{\sqrt{2 \pi}} \frac{3 \sigma_{t}^{2} s-s^{3}}{\sigma_{t}^{7}} \exp \left(-\frac{s^{2}}{2 \sigma_{t}^{2}}\right) .
\end{aligned}
$$

As $s=-\sigma_{t}$ (the solution to $f_{t}^{\prime \prime}(s)=0$ and $f_{t}^{\prime \prime \prime}(s)<0$ ) maximizes $f_{t}^{\prime}(x)$, we obtain $\forall x \in \mathbb{R} f_{t}^{\prime}(x) \leq-\frac{1}{\sqrt{2 \pi}} \frac{-\sigma_{t}}{\sigma_{t}^{3}} \exp \left(-\frac{\sigma_{t}^{2}}{2 \sigma_{t}^{2}}\right)$ and thus

$$
f_{t}^{\prime}(x) \leq \frac{1}{\sigma_{t}^{2} \sqrt{2 \pi \exp (1)}}
$$

Furthermore, (30) implies

$$
\int_{0}^{\infty} f_{2}(\eta s) d s=\frac{1}{\sigma_{2} \sqrt{2 \pi}} \int_{0}^{\infty} \exp \left(-\left(\frac{s \eta}{\sqrt{2} \sigma_{2}}\right)^{2}\right) d s
$$

Substituting $s=\frac{\sqrt{2} \sigma_{2}}{\eta} t$ and $d s=\frac{\sqrt{2} \sigma_{2}}{\eta} d t$ implies

$$
\int_{0}^{\infty} f_{2}(\eta s) d s=\frac{1}{\eta \sqrt{\pi}} \int_{0}^{\infty} \exp \left(-t^{2}\right) d t
$$

With (29), we get

$$
\int_{0}^{\infty} f_{2}(\eta s) d s=\frac{1}{2 \eta} .
$$


Next, (30) and (31) imply

$$
\int_{0}^{\infty} f_{2}(\eta s) f_{2}^{\prime}(\eta s) d s=-\frac{\eta}{2 \pi \sigma_{2}^{4}} \int_{0}^{\infty} s \cdot \exp \left(-\frac{s^{2} \eta^{2}}{\sigma_{2}^{2}}\right) d s .
$$

Substituting $s=\frac{\sqrt{t} \sigma_{2}}{\eta}$ and $d s=\frac{\sigma_{2}}{2 \sqrt{t} \eta} d t$ and noting that $\int_{0}^{\infty} \exp (-t) d t=1$, we obtain

$$
\int_{0}^{\infty} f_{2}(\eta s) f_{2}^{\prime}(\eta s) d s=-\frac{1}{4 \pi \eta \sigma_{2}^{2}}
$$

Furthermore, (30) implies

$$
C(\eta)=\frac{1}{\pi \sigma_{1} \sigma_{2}} \int_{0}^{\infty} \exp \left(-\left(s \frac{\sqrt{\sigma_{1}^{2} \eta^{2}+\sigma_{2}^{2}}}{\sqrt{2} \sigma_{1} \sigma_{2}}\right)^{2}\right) d s
$$

Substituting $s=\frac{\sqrt{2} \sigma_{1} \sigma_{2}}{\sqrt{\sigma_{1}^{2} \eta^{2}+\sigma_{2}^{2}}} t$ and $d s=\frac{\sqrt{2} \sigma_{1} \sigma_{2}}{\sqrt{\sigma_{1}^{2} \eta^{2}+\sigma_{2}^{2}}} d t$ yields

$$
C(\eta)=\frac{\sqrt{2}}{\pi \sqrt{\sigma_{1}^{2} \eta^{2}+\sigma_{2}^{2}}} \int_{0}^{\infty} \exp \left(-t^{2}\right) d t
$$

With (29), we get

$$
C(\eta)=\frac{1}{\sqrt{2 \pi} \sqrt{\sigma_{1}^{2} \eta^{2}+\sigma_{2}^{2}}}
$$

so that

$$
C^{\prime}(\eta)=-\frac{\eta \sigma_{1}^{2}}{\sqrt{2 \pi}\left(\sigma_{1}^{2} \eta^{2}+\sigma_{2}^{2}\right)^{\frac{3}{2}}} .
$$

\section{Part 2: Second-Order Conditions}

Next, we derive sufficient conditions for the second-order conditions to hold. ${ }^{41}$ Using $K_{t}^{\prime \prime}\left(e_{i t}\right)=k,(19)$ simplifies to

$$
f_{2}^{\prime}(x) W_{2}<k \forall x \in \mathbb{R}
$$

From $W_{2} \leq W$ and (32),

$$
f_{2}^{\prime}(x) W_{2} \leq \frac{W}{\sigma_{2}^{2} \sqrt{2 \pi \exp (1)}}
$$

\footnotetext{
${ }^{41}$ We only consider the second-order conditions for the full revelation case.
} 
(37) and (38) imply that a sufficient condition for (19) to hold is

$$
k>\frac{W}{\sigma_{2}^{2} \sqrt{2 \pi \exp (1)}}
$$

Similarly, (28) can be written as

$$
\begin{gathered}
f_{1}^{\prime}(x) W_{1}+\eta W_{2} \int_{0}^{\infty} f_{2}(\eta s)\left[f_{1}^{\prime}(s+x)-f_{1}^{\prime}(s-x)\right] d s \\
+\frac{\eta W_{2}^{2}}{k} \int_{0}^{\infty} f_{2}(\eta s) f_{2}^{\prime}(\eta s)\left[f_{1}^{\prime}(s+x)+f_{1}^{\prime}(s-x)\right] d s<k
\end{gathered}
$$

Using (32), we obtain $\forall x \in \mathbb{R}$

$$
\begin{gathered}
f_{1}^{\prime}(x) W_{1} \leq \frac{W_{1}}{\sigma_{1}^{2} \sqrt{2 \pi \exp (1)}} \\
\eta W_{2} \int_{0}^{\infty} f_{2}(\eta s)\left[f_{1}^{\prime}(s+x)-f_{1}^{\prime}(s-x)\right] d s \leq \frac{2 W_{2} \eta \int_{0}^{\infty} f_{2}(\eta s) d s}{\sigma_{1}^{2} \sqrt{2 \pi \exp (1)}} \\
\frac{\eta W_{2}^{2}}{k} \int_{0}^{\infty} f_{2}(\eta s) f_{2}^{\prime}(\eta s)\left[f_{1}^{\prime}(s+x)+f_{1}^{\prime}(s-x)\right] d s \leq \frac{2 W_{2}^{2}\left|\eta \int_{0}^{\infty} f_{2}(\eta s) f_{2}^{\prime}(\eta s) d s\right|}{k \sigma_{1}^{2} \sqrt{2 \pi \exp (1)}} .
\end{gathered}
$$

This yields an upper bound for the left-hand side of (40):

$$
\frac{1}{\sigma_{1}^{2} \sqrt{2 \pi \exp (1)}}\left[W_{1}+2 W_{2} \eta \int_{0}^{\infty} f_{2}(\eta s) d s+\frac{2 W_{2}^{2}}{k}\left|\eta \int_{0}^{\infty} f_{2}(\eta s) f_{2}^{\prime}(\eta s) d s\right|\right] .
$$

With (33) and (34), this upper bound can be written as

$$
\frac{W_{1}+W_{2}}{\sigma_{1}^{2} \sqrt{2 \pi \exp (1)}}+\frac{W_{2}^{2}}{k \sigma_{1}^{2} \sigma_{2}^{2}(2 \pi)^{\frac{3}{2}} \sqrt{\exp (1)}} \leq \frac{W}{\sigma_{1}^{2} \sqrt{2 \pi \exp (1)}}+\frac{W^{2}}{k \sigma_{1}^{2} \sigma_{2}^{2}(2 \pi)^{\frac{3}{2}} \sqrt{\exp (1)}} .
$$

A sufficient condition for (28) to hold is thus

$$
k>\frac{W}{\sigma_{1}^{2} \sqrt{2 \pi \exp (1)}}+\frac{W^{2}}{k \sigma_{1}^{2} \sigma_{2}^{2}(2 \pi)^{\frac{3}{2}} \sqrt{\exp (1)}} .
$$

\section{Part 3: Characterizing the equilibrium}

By Part 2, Proposition 1 characterizes the PBE. Inserting $\left(K_{t}^{\prime}\right)^{-1}(x)=\frac{x}{k}$, (30) and (35) in (9) and (10) yields (11) and (12). 


\subsection{Prizes vs. Weights: An illustration}

\subsubsection{Proof of Observation 1}

(i) The statement for the pure prize policy with $W_{1}=W$ follows directly from Corollary 3. The result for $e_{1}$ and $\eta \rightarrow \infty$ follows from $\lim _{\eta \rightarrow \infty} \eta / \sqrt{\sigma_{2}^{2}+\sigma_{1}^{2} \eta^{2}}=\lim _{\eta \rightarrow \infty} 1 / \sqrt{\sigma_{1}^{2}+\sigma_{2}^{2} / \eta^{2}}=1 / \sigma_{1}$ and (11). The result for for $e_{2}$ and $\eta \rightarrow \infty$ follows from (12). (ii) These two policies are equivalent by definition. The result follows from Corollary 3 (ii).

\subsubsection{Proof of Observation 2}

By Corollary 3, a pure prize policy $\left(W_{1}, W-W_{1}\right)$ induces first-period efforts $W_{1} / k \sigma_{1} \sqrt{2 \pi}$ and expected second-period efforts $\left(W-W_{1}\right) / k \sigma_{2} \sqrt{2 \pi}$. The result on prize policies thus follows from $\sigma_{1}=\sigma_{2}=\sigma$. A pure weight policy with $W_{2}=W$ and weight $\eta$ induces first-period efforts $\eta W / k \sqrt{2 \pi}\left(\sqrt{\sigma_{2}^{2}+\sigma_{1}^{2} \eta^{2}}\right)$ and expected second-period efforts $W / k \sqrt{2 \pi} \sqrt{\sigma_{2}^{2}+\sigma_{1}^{2} \eta^{2}}$, which implies the result for pure weight policies.

\subsection{Results for Perfect Substitutes}

We now prove the results discussed in Subsections 6.1 and 7.1.1. In Lemma 5, we derive the optimal prize structure (conditional on the weight $\eta$ ) for the case that $K_{1}$ and $K_{2}$ are not necessarily quadratic (as discussed in Subsection 7.1.1). The result will rely on the Assumption that $K_{t}^{\prime \prime \prime} \leq 0 .{ }^{42}$ Then, we will use Lemma 5 to prove the results discussed in Subsection 6.1. We will first show how Proposition 2 for $K_{t}^{\prime \prime \prime}=0$ follows from Lemma 5 and then derive Corollary 4.

In the following, for perfect substitutes, we will denote the optimal choice of $\eta$ conditional on $W_{1}$ and $\rho$ as $\eta^{P}\left(W_{1}, \rho\right)$ and the optimal choice of $W_{1}$ conditional on $\eta$ as $W_{1}^{P}(\eta, \rho)$.

\footnotetext{
${ }^{42}$ This is not a serious restriction: Corollary 6 in Section 8 will show that $K_{2}^{\prime \prime \prime} \leq 0$ is the case in which it is optimal to give feedback.
} 


\subsubsection{General costs}

Lemma 5 Suppose $K_{t}^{\prime \prime \prime} \leq 0$ for $t=1$, 2 . For $\eta>0, W_{1}^{P}(\eta, 1)=0\left(W_{1}^{P}(\eta, 1)=W\right)$ if and only if

$W f_{1}(0)<(>) K_{1}^{\prime}\left[\left(K_{1}^{\prime}\right)^{-1}(\eta W C(\eta))+2 \int_{0}^{\infty}\left(K_{2}^{\prime}\right)^{-1}\left(f_{2}(\eta s) W\right) f_{1}(s) d s\right]$.

Proof. Using (9) and (10) in (13) gives

$$
\begin{aligned}
V^{P}\left(\eta, W_{1}, 1\right) & =\left(K_{1}^{\prime}\right)^{-1}\left(f_{1}(0) W_{1}+\eta\left(W-W_{1}\right) C(\eta)\right) \\
& +2 \int_{0}^{\infty}\left(K_{2}^{\prime}\right)^{-1}\left(f_{2}(\eta s)\left(W-W_{1}\right)\right) f_{1}(s) d s
\end{aligned}
$$

This yields

$$
\begin{gathered}
\frac{\partial V^{P}\left(\eta, W_{1}, 1\right)}{\partial W_{1}}=\frac{f_{1}(0)-\eta C(\eta)}{K_{1}^{\prime \prime}\left[\left(K_{1}^{\prime}\right)^{-1}\left(f_{1}(0) W_{1}+\eta\left(W-W_{1}\right) C((\eta))\right]\right.} \\
-2 \int_{0}^{\infty} \frac{f_{2}(\eta s) f_{1}(s)}{K_{2}^{\prime \prime}\left[\left(K_{2}^{\prime}\right)^{-1}\left(f_{2}(\eta s)\left(W-W_{1}\right)\right)\right]} d s
\end{gathered}
$$

and hence

$$
\begin{gathered}
\frac{\partial^{2} V^{P}\left(\eta, W_{1}, 1\right)}{\partial W_{1}^{2}}= \\
-\frac{K_{1}^{\prime \prime \prime}\left[\left(K_{1}^{\prime}\right)^{-1}\left(f_{1}(0) W_{1}+\eta\left(W-W_{1}\right) C(\eta)\right)\right]\left(f_{1}(0)-\eta C(\eta)\right)^{2}}{\left(K_{1}^{\prime \prime}\left[\left(K_{1}^{\prime}\right)^{-1}\left(f_{1}(0) W_{1}+\eta\left(W-W_{1}\right) C(\eta)\right)\right]\right)^{3}} \\
-2 \int_{0}^{\infty} \frac{\left(f_{2}(\eta s)\right)^{2} K_{2}^{\prime \prime \prime}\left[\left(K_{2}^{\prime}\right)^{-1}\left(f_{2}(\eta s)\left(W-W_{1}\right)\right)\right] f_{1}(s) d s}{\left(K_{2}^{\prime \prime}\left[\left(K_{2}^{\prime}\right)^{-1}\left(f_{2}(\eta s)\left(W-W_{1}\right)\right)\right]\right)^{3}} .
\end{gathered}
$$

Since $K_{t}^{\prime \prime}>0, K_{t}^{\prime \prime \prime} \leq 0$ implies $\frac{\partial^{2} V^{P}\left(\eta, W_{1}, 1\right)}{\left(\partial W_{1}\right)^{2}} \geq 0$. Thus, there is no interior optimum. For $W_{1}=0$ and $W_{1}=W$, the principal's expected payoffs are

$$
\begin{gathered}
V^{P}(\eta, 0,1)=\left(K_{1}^{\prime}\right)^{-1}(\eta W C(\eta))+2 \int_{0}^{\infty}\left(K_{2}^{\prime}\right)^{-1}\left(f_{2}(\eta s) W\right) f_{1}(s) d s \\
V^{P}(\eta, W, 1)=\left(K_{1}^{\prime}\right)^{-1}\left(f_{1}(0) W\right) .
\end{gathered}
$$


Therefore,

$$
\begin{gathered}
V^{P}(\eta, 0,1)-V^{P}(\eta, W, 1)= \\
\left(K_{1}^{\prime}\right)^{-1}(\eta W C(\eta))+2 \int_{0}^{\infty}\left(K_{2}^{\prime}\right)^{-1}\left(f_{2}(\eta s) W\right) f_{1}(s) d s-\left(K_{1}^{\prime}\right)^{-1}\left(f_{1}(0) W\right) .
\end{gathered}
$$

Hence, $V^{P}(\eta, 0,1)-V^{P}(\eta, W, 1)>(<) 0$ if and only if (42) holds.

\subsubsection{Quadratic Costs: Proof of Proposition 2}

The proof relies heavily on the following result:

Lemma 6 Suppose $W_{1}<W$. Then,

$$
\begin{aligned}
& \text { (i) } \partial e_{1}^{*}\left(\eta, W_{1}, W-W_{1}\right) /\left.\partial \eta\right|_{\eta=0}>0 . \\
& \text { (ii) } \partial E\left(e_{2}^{*}\left(\eta, W-W_{1}, 1\right)\right) /\left.\partial \eta\right|_{\eta=0}=0 .
\end{aligned}
$$

Proof. (i) From (9),

$$
\frac{\partial e_{1}^{*}\left(\eta, W_{1}, W-W_{1}\right)}{\partial \eta}=\frac{\left(W-W_{1}\right)\left(C(\eta)+\eta C^{\prime}(\eta)\right)}{K_{1}^{\prime \prime}\left[\left(K_{1}^{\prime}\right)^{-1}\left(f_{1}(0) W_{1}+\eta\left(W-W_{1}\right) C(\eta)\right)\right]} .
$$

Hence,

$$
\begin{aligned}
\left.\frac{\partial e_{1}^{*}\left(\eta, W_{1}, W-W_{1}\right)}{\partial \eta}\right|_{\eta=0} & =\frac{\left(W-W_{1}\right) C(0)}{K_{1}^{\prime \prime}\left[\left(K_{1}^{\prime}\right)^{-1}\left(f_{1}(0) W_{1}\right)\right]} \\
& =\frac{\left(W-W_{1}\right) f_{2}(0)}{K_{1}^{\prime \prime}\left[\left(K_{1}^{\prime}\right)^{-1} f_{1}(0) W_{1}\right]} .
\end{aligned}
$$

where the second equality follows from (8(ii)). As $K_{1}^{\prime \prime}>0$ and $f_{2}(0)>0$, $\left.\frac{\partial e_{1}^{*}\left(\eta, W_{1}, W-W_{1}\right)}{\partial \eta}\right|_{\eta=0}>0$ provided $W_{1}<W$.

(ii) From (10),

$$
\frac{\partial E\left(e_{2}^{*}\left(\eta, W-W_{1}, 1\right)\right)}{\partial \eta}=2 \int_{0}^{\infty} \frac{s f_{2}^{\prime}(\eta s)\left(W-W_{1}\right) f_{1}(s)}{K_{2}^{\prime \prime}\left[\left(K_{2}^{\prime}\right)^{-1}\left(f_{2}(\eta s)\left(W-W_{1}\right)\right)\right]} d s .
$$


Hence,

$$
\left.\frac{\partial E\left(e_{2}^{*}\left(\eta, W-W_{1}, 1\right)\right)}{\partial \eta}\right|_{\eta=0}=2 \int_{0}^{\infty} \frac{s f_{2}^{\prime}(0)\left(W-W_{1}\right) f_{1}(s)}{K_{2}^{\prime \prime}\left[\left(K_{2}^{\prime}\right)^{-1}\left(f_{2}(0)\left(W-W_{1}\right)\right)\right]} d s=0,
$$

where the second equality follows from $f_{2}^{\prime}(0)=0$.

We now prove the proposition:

(i) With $K_{t}^{\prime \prime \prime}=0$, Lemma 5 implies that $W_{1}^{P}(\eta)$ always is a boundary solution, with $W_{1}^{P}(\eta)=0$ if $\frac{1}{k_{1}} f_{1}(0)<\left(\frac{\eta}{k_{1}}+\frac{1}{k_{2}}\right) C(\eta)$. Hence, if the inequality is satisfied for some $\eta$, then total effort for this $\eta$ and $W_{1}=0$ is higher than for $W_{1}=W$, which shows that $W_{1}=W$ cannot be optimal. In this case, since there is no interior optimum by Lemma $5, W_{1}=0$ is optimal. ${ }^{43}$

From (13),

$$
\frac{\partial V^{P}\left(\eta, W_{1}, 1\right)}{\partial \eta}=\frac{\partial e_{1}^{*}\left(\eta, W_{1}, W-W_{1}\right)}{\partial \eta}+\frac{\partial E\left(e_{2}^{*}\left(\eta_{1}, W-W_{1}, 1\right)\right)}{\partial \eta}
$$

Using (C1) and (7) to simplify (44) and (45), (46) becomes

$$
\frac{\partial V^{P}\left(\eta, W_{1}\right)}{\partial \eta}=\left(W-W_{1}\right)\left(\frac{C(\eta)+\eta C^{\prime}(\eta)}{k_{1}}+\frac{C^{\prime}(\eta)}{k_{2}}\right)
$$

Solving $\frac{\partial V^{P}\left(\eta, W_{1}\right)}{\partial \eta}=0$ and rearranging gives (14).

(ii) follows from reverting the argument from (i) that $W_{1}=0$ if $\frac{1}{k_{1}} f_{1}(0)<\left(\frac{\eta}{k_{1}}+\frac{1}{k_{2}}\right) C(\eta)$.

\subsubsection{Normal-Quadratic Example: Proof of Corollary 4}

We first derive $\eta^{P}\left(W_{1}\right)$. With (35) and (36), we obtain

$$
\frac{C^{\prime}(\eta)}{C(\eta)}=-\frac{\eta \sigma_{1}^{2}}{\sigma_{2}^{2}+\sigma_{1}^{2} \eta^{2}}
$$

Proposition 2 (i) thus implies $\frac{\eta \cdot \sigma_{1}^{2}}{\sigma_{2}^{2}+\sigma_{1}^{2} \eta^{2}}=\frac{1}{1+\eta}$ as a necessary condition, which is uniquely (and positively) solved by $\eta=\frac{\sigma_{2}^{2}}{\sigma_{1}^{2}}>0$. Since the optimal $\eta$ must be strictly positive by Lemma 6 and since the solution to the necessary condition is unique and positive, the necessary condition is sufficient and we have $\eta^{P}\left(W_{1}\right)=\frac{\sigma_{2}^{2}}{\sigma_{1}^{2}} \forall W_{1}<W$. Next, we show

\footnotetext{
${ }^{43}$ This can also be derived from Proposition 8.
} 
that $W_{1}^{P}=0$. By Corollary $2, W_{1}^{P}=0$ if $\exists \eta$ such that $f_{1}(0)<(1+\eta) C(\eta)$. From (30) and (35), this condition is equivalent with

$\sqrt{2 \pi} \sqrt{\sigma_{1}^{2} \eta^{2}+\sigma_{2}^{2}}<\sigma_{1} \sqrt{2 \pi}(1+\eta) \Longleftrightarrow 2 \eta>\sigma_{2}^{2} / \sigma_{1}^{2}-1$. In particular, this holds for $\eta^{P}\left(W_{1}\right)=\sigma_{2}^{2} / \sigma_{1}^{2}$. Hence, $W_{1}^{P}=0$ and $\eta^{P}=\eta^{P}\left(W_{1}^{P}\right)=\sigma_{2}^{2} / \sigma_{1}^{2}$.

\subsection{Results for Imperfect Substitutes}

We now prove the results discussed in Subsection 6.2. After some preliminary calculation for the case of general cost functions, we prove Proposition 3 for the quadratic case. Finally, we deal with the normal-quadratic example. For imperfect substitutes, we denote the optimal choice of $\eta$ conditional on $W_{1}$ and $\rho$ as $\eta^{I}\left(W_{1}, \rho\right)$ and the optimal choice of $W_{1}$ conditional on $\eta$ as $W_{1}^{I}(\eta, \rho)$.

\subsubsection{General Costs: Preliminary Calculations}

The expected payoff of the principal is

$$
V^{I}\left(\eta_{1}, W_{1}, 1\right)=e_{1}^{*}\left(\eta_{1}, W-W_{1}, 1\right) \cdot E\left(e_{2}^{*}\left(\eta_{2}, W-W_{1}, 1\right)\right) .
$$

Using (9) and (10) in (48) yields

$$
\begin{aligned}
V^{I}\left(\eta_{1}, W_{1}, 1\right) & =\left(K_{1}^{\prime}\right)^{-1}\left(f_{1}(0) W_{1}+\eta\left(W-W_{1}\right) C(\eta)\right) \\
& 2 \int_{0}^{\infty}\left(K_{2}^{\prime}\right)^{-1}\left(f_{2}(\eta s)\left(W-W_{1}\right)\right) f_{1}(s) d s
\end{aligned}
$$

Using (49), we have

$$
\begin{gathered}
\frac{\partial V^{I}\left(\eta, W_{1}, 1\right)}{\partial W_{1}}= \\
\frac{2\left(f_{1}(0)-\eta C(\eta)\right) \int_{0}^{\infty}\left(K_{2}^{\prime}\right)^{-1}\left(f_{2}(\eta s)\left(W-W_{1}\right)\right) f_{1}(s) d s}{K_{1}^{\prime \prime}\left[\left(K_{1}^{\prime}\right)^{-1}\left(f_{1}(0) W_{1}+\eta\left(W-W_{1}\right) C(\eta)\right)\right]} \\
-2\left(K_{1}^{\prime}\right)^{-1}\left(f_{1}(0) W_{1}+\eta\left(W-W_{1}\right) C(\eta)\right) \cdot \\
\int_{0}^{\infty} \frac{f_{2}(\eta s)}{K_{2}^{\prime \prime}\left[\left(K_{2}^{\prime}\right)^{-1}\left(f_{2}(\eta s)\left(W-W_{1}\right)\right)\right]} f_{1}(s) d s .
\end{gathered}
$$


Thus,

$$
\begin{gathered}
\frac{\partial^{2} V^{I}\left(\eta, W_{1}, 1\right)}{\left(\partial W_{1}\right)^{2}}= \\
-\frac{2\left(f_{1}(0)-\eta C(\eta)\right)^{2} \cdot K_{1}^{\prime \prime \prime}\left[\left(K_{1}^{\prime}\right)^{-1}\left(f_{1}(0) W_{1}+\eta\left(W-W_{1}\right) C(\eta)\right)\right]}{\left(K_{1}^{\prime \prime}\left[\left(K_{1}^{\prime}\right)^{-1}\left(f_{1}(0) W_{1}+\eta\left(W-W_{1}\right) C(\eta)\right)\right]\right)^{3}} \\
\int_{0}^{\infty}\left(K_{2}^{\prime}\right)^{-1}\left(f_{2}(\eta s)\left(W-W_{1}\right)\right) f_{1}(s) d s \\
-\frac{\infty\left(f_{1}(0)-\eta C(\eta)\right)}{K_{1}^{\prime \prime}\left[\left(K_{1}^{\prime}\right)^{-1}\left(f_{1}(0) W_{1}+\eta\left(W-W_{1}\right) C(\eta)\right)\right]} \int_{0}^{\infty} \frac{f_{2}(\eta s) f_{1}(s) d s}{K_{2}^{\prime \prime}\left[\left(K_{2}^{\prime}\right)^{-1}\left(f_{2}(\eta s)\left(W-W_{1}\right)\right)\right]} \\
-\left(K_{1}^{\prime}\right)^{-1}\left(f_{1}(0) W_{1}+\eta\left(W-W_{1}\right) C(\eta)\right) \cdot 2 \int_{0}^{\infty} \frac{\left(f_{2}(\eta s)\right)^{2}}{\left(K_{2}^{\prime \prime}\left[\left(K_{2}^{\prime}\right)^{-1}\left(f_{2}(\eta s)\left(W-W_{1}\right)\right)\right]\right)^{3}} \\
K_{2}^{\prime \prime \prime}\left[\left(K_{2}^{\prime}\right)^{-1}\left(f_{2}(\eta s)\left(W-W_{1}\right)\right)\right] f_{1}(s) d s .
\end{gathered}
$$

\subsubsection{Proof of Proposition 3}

The proof requires an auxilliary result.

Lemma 7 Suppose (C1) holds. For all $\eta>0, W_{1}^{I}(\eta)>0$ if and only if $f_{1}(0)>2 \eta C(\eta)$. In this case

$$
W_{1}^{I}(\eta)=W \frac{f_{1}(0)-2 \eta C(\eta)}{2 f_{1}(0)-2 \eta C(\eta)}>0
$$

Proof. Clearly $W_{1}>0$ at the optimum if $\left.\frac{\partial V^{I}\left(\eta, W_{1}\right)}{\partial W_{1}}\right|_{W_{1}=0}>0$, that is, using (53), if $f_{1}(0)>2 \eta C(\eta)$. To see that $W_{1}=0$ at the optimum if $\left.\frac{\partial V^{I}\left(\eta, W_{1}\right)}{\partial W_{1}}\right|_{W_{1}=0}<0$ or, equivalently, $f_{1}(0)<2 \eta C(\eta)$, first note that (53) implies

$$
\frac{\partial^{2} V^{I}\left(\eta, W_{1}\right)}{\partial W_{1}^{2}}=\frac{C(\eta)}{k_{1} k_{2}}\left[-2 f_{1}(0)+2 \eta C(\eta)\right] .
$$

Thus $\frac{\partial V^{I}\left(\eta, W_{1}\right)}{\partial W_{1}}$ is monotone in $W_{1}$.

With (C1), (49) yields

$$
V^{I}\left(\eta, W_{1}\right)=\frac{\left(W-W_{1}\right) C(\eta)}{k_{1} k_{2}}\left(f_{1}(0) W_{1}+\eta\left(W-W_{1}\right) C(\eta)\right) .
$$


Thus

$$
\frac{\partial V^{I}\left(\eta, W_{1}\right)}{\partial W_{1}}=\frac{C(\eta)}{k_{1} k_{2}}\left[f_{1}(0)\left(W-2 W_{1}\right)-2 \eta C(\eta)\left(W-W_{1}\right)\right]
$$

and $\frac{\partial V^{I}\left(\eta, W_{1}\right)}{\partial W_{1}}<0 \forall W_{1}>\frac{W}{2}$. The last two statements imply that, whenever $\left.\frac{\partial V^{I}\left(\eta, W_{1}\right)}{\partial W_{1}}\right|_{W_{1}=0}<0$, then $\frac{\partial V^{I}\left(\eta, W_{1}\right)}{\partial W_{1}}<0$ for all $W_{1} \leq \frac{W}{2}$ and thus $W_{1}^{I}(\eta)=0$. To see that $W_{1}=0$ at the optimum if $\left.\frac{\partial V^{I}\left(\eta, W_{1}\right)}{\partial W_{1}}\right|_{W_{1}=0}=0$ or, equivalently, $f_{1}(0)=2 \eta C(\eta)$, note that $f_{1}(0)=2 \eta C(\eta)$ implies $\frac{\partial^{2} V^{I}\left(\eta, W_{1}\right)}{\partial W_{1}^{2}}<0$, so that $\frac{\partial V^{I}\left(\eta, W_{1}\right)}{\partial W_{1}}<0 \forall W_{1}>0$ and thus $W_{1}^{I}(\eta)=0$. For $\left.\frac{\partial V^{I}\left(\eta, W_{1}\right)}{\partial W_{1}}\right|_{W_{1}=0}>0$, the first-order condition $\frac{\partial V^{I}\left(\eta, W_{1}\right)}{\partial W_{1}}=0$ yields $W_{1}^{I}(\eta)=W \frac{f_{1}(0)-2 \eta C(\eta)}{2 f_{1}(0)-2 \eta C(\eta)}>0$ for $f_{1}(0)>2 \eta C(\eta)$. Summing up, we obtain

$$
W_{1}^{I}(\eta)=\left\{\begin{array}{cl}
W \frac{f_{1}(0)-2 \eta C(\eta)}{2 f_{1}(0)-2 \eta C(\eta)}>0, & f_{1}(0)>2 \eta C(\eta) \\
0, & f_{1}(0) \leq 2 \eta C(\eta)
\end{array}\right.
$$

We can now prove Part (i) of Proposition 3. (54) shows that $W_{1}^{I}$ must correspond to one of the two cases mentioned in Proposition 3. To complete the proof, we derive the first-order condition for $\eta^{I}\left(W_{1}\right)$ for these two cases. From (49), we obtain

$$
\begin{gathered}
\frac{\partial V^{I}\left(\eta, W_{1}\right)}{\partial \eta}=\frac{\left(W-W_{1}\right)^{2} C(\eta)}{k_{1} k_{2}}\left(C(\eta)+\eta C^{\prime}(\eta)\right)+ \\
\frac{\left(W-W_{1}\right) C^{\prime}(\eta)}{k_{1} k_{2}}\left(f_{1}(0) W_{1}+\eta\left(W-W_{1}\right) C(\eta)\right) .
\end{gathered}
$$

The first-order condition $\frac{\partial V^{I}\left(\eta, W_{1}\right)}{\partial \eta}=0$ yields

$$
W_{1}=W \frac{(C(\eta))^{2}+2 \eta C(\eta) C^{\prime}(\eta)}{C(\eta)^{2}+2 \eta C(\eta) C^{\prime}(\eta)-f_{1}(0) C^{\prime}(\eta)} .
$$

According to Lemma 7, $W_{1}=0$ is a necessary condition for an optimum with $f_{1}(0) \leq$ $2 \eta C(\eta)$. Inserting $W_{1}=0$ in (56) gives the first-order condition $\eta=-\frac{C(\eta)}{2 C^{\prime}(\eta)}$, which corresponds to Proposition 3(a). Analogously, $W_{1}=W \frac{f_{1}(0)-2 \eta C(\eta)}{2 f_{1}(0)-2 \eta C(\eta)}$ is a necessary condition for an optimum with $f_{1}(0)>2 \eta C(\eta)$ Inserting $W_{1}=W \frac{f_{1}(0)-2 \eta C(\eta)}{2 f_{1}(0)-2 \eta C(\eta)}$ in $(56)$ and solving for $f_{1}(0)$ gives the first-order condition $f_{1}(0)=-\frac{(C(\eta))^{2}}{C^{\prime}(\eta)}$, 
which corresponds to Proposition 3(b).

\subsubsection{Normal-Quadratic Example: Proof of Corollary 5}

(35) and (36) yield

$$
C(\eta)^{2}+C^{\prime}(\eta) f_{1}(0)=\frac{\sqrt{\eta^{2} \sigma_{1}^{2}+\sigma_{2}^{2}}-\eta \sigma_{1}}{2 \pi\left(\eta^{2} \sigma_{1}^{2}+\sigma_{2}^{2}\right)^{\frac{3}{2}}}>0 \forall \eta .
$$

This is inconsistent with $\left|C^{\prime}(\eta) / C(\eta)\right|=\left|C(\eta) / f_{1}(0)\right|$ as for $\eta>0,\left|C^{\prime}(\eta) / C(\eta)\right|=$ $\left|C(\eta) / f_{1}(0)\right|$ is equivalent to $C(\eta)^{2}+C^{\prime}(\eta) f_{1}(0)=0$. Therefore, according to Proposition 3(ii)(b), $W_{1}^{I}>0$ cannot apply. Hence, Proposition (3)(ii) gives $\left|C^{\prime}(\eta) / C(\eta)\right|=1 / 2 \eta$ as the necessary condition for $\eta^{I}$. Using (35) and (36), this can be written as $\left|\eta \sigma_{1}^{2} / \sigma_{2}^{2}+\sigma_{1}^{2} \eta^{2}\right|=$ $1 / 2 \eta$, which is solved by $\eta^{I}=\sigma_{2} / \sigma_{1}$.

\subsection{The role of feedback}

We now provide additional material concerning Section 8. We prove the equilibrium characterization for the no feedback case, Proposition 5. We then discuss second-order conditions. Next we prove Lemma 3, which compares the policies with and without feedback. Finally, we characterize the optimal prize policy in the absence of feedback.

\subsubsection{Proof of Proposition 5}

(i) From (16), the first-order conditions are

$$
\begin{array}{r}
f_{1}\left(\Delta e_{i 1}\right) W_{1}+\eta W_{2} \int_{-\infty}^{\infty} f_{2}\left(\eta\left(\Delta e_{i 1}+s\right)+\Delta e_{i 2}\right) f_{1}(s) d s=K_{1}^{\prime}\left(e_{i 1}\right) \\
W_{2} \int_{-\infty}^{\infty} f_{2}\left(\eta\left(\Delta e_{i 1}+s\right)+\Delta e_{i 2}\right) f_{1}(s) d s=K_{2}^{\prime}\left(e_{i 2}\right)
\end{array}
$$

For the symmetric case $\Delta e_{i 1}=\Delta e_{i 2}=0$, this simplifies to

$$
\begin{aligned}
f_{1}(0) W_{1}+\eta W_{2} C(\eta) & =K_{1}^{\prime}\left(e_{i 1}\right) ; \\
W_{2} C(\eta) & =K_{2}^{\prime}\left(e_{i 2}\right) .
\end{aligned}
$$


Inverting $K_{1}^{\prime}$ and $K_{2}^{\prime}$ yields (17) and (18).

(ii) First-period equilibrium efforts are positive because $\eta \geq 0$ and $K_{1}^{\prime}(0)=0$ by Assumption 1. Equilibrium efforts in the second period are positive because $W_{2} C(\eta)>0$ by Assumption 2. By part (i), (17) and (18) are necessary equilibrium conditions.

Consider the following second-order conditions ${ }^{44}$

$$
\begin{gathered}
f_{1}^{\prime}\left(\Delta e_{i 1}\right) W_{1}+\eta^{2} W_{2} \int_{-\infty}^{\infty} f_{2}^{\prime}\left(\eta\left(\Delta e_{i 1}+s\right)+\Delta e_{i 2}\right) f_{1}(s) d s<K_{1}^{\prime \prime}\left(e_{i 1}\right) ; \quad(57) \\
K_{1}^{\prime \prime}\left(e_{i 1}\right) W_{2} \int_{-\infty}^{\infty} f_{2}^{\prime}\left(\eta\left(\Delta e_{i 1}+s\right)+\Delta e_{i 2}\right) f_{1}(s) d s \\
+K_{2}^{\prime \prime}\left(e_{i 2}\right) \cdot\left[f_{1}^{\prime}\left(\Delta e_{i 1}\right) W_{1}+\eta^{2} W_{2} \int_{-\infty}^{\infty} f_{2}^{\prime}\left(\eta\left(\Delta e_{i 1}+s\right)+\Delta e_{i 2}\right) f_{1}(s) d s\right] \\
-f_{1}^{\prime}\left(\Delta e_{i 1}\right) W_{1} W_{2} \int_{-\infty}^{\infty} f_{2}^{\prime}\left(\eta\left(\Delta e_{i 1}+s\right)+\Delta e_{i 2}\right) f_{1}(s) d s<K_{1}^{\prime \prime}\left(e_{i 1}\right) K_{2}^{\prime \prime}\left(e_{i 2}\right) .
\end{gathered}
$$

If these conditions hold globally, the expected payoff of player $i$ is a strictly concave function of $\left(e_{i 1}, e_{i 2}\right)$, so that (17) and (18) describe best responses, and thus characterize a Nash equilibrium. Furthermore, this is the unique symmetric equilibrium.

\subsubsection{Discussing Second-Order Conditions (No feedback)}

Global Second-Order Conditions We first show that (57) and (58) hold for given policy parameters and distributions as long as $K_{t}$ is sufficiently convex for $t=1,2$. For (57), this is obvious, as the right-hand side is increasing in $K_{1}^{\prime \prime}()$. To see that the statement is also true for (58), let

$$
\begin{aligned}
& A \equiv W_{2} \int_{-\infty}^{\infty} f_{2}^{\prime}\left(\eta\left(\Delta e_{i 1}+s\right)+\Delta e_{i 2}\right) f_{1}(s) d s \\
& B \equiv f_{1}^{\prime}\left(\Delta e_{i 1}\right) W_{1}+\eta^{2} W_{2} \int_{-\infty}^{\infty} f_{2}^{\prime}\left(\eta\left(\Delta e_{i 1}+s\right)+\Delta e_{i 2}\right) f_{1}(s) d s \\
& C \equiv-f_{1}^{\prime}\left(\Delta e_{i 1}\right) W_{1} W_{2} \int_{-\infty}^{\infty} f_{2}^{\prime}\left(\eta\left(\Delta e_{i 1}+s\right)+\Delta e_{i 2}\right) f_{1}(s) d s
\end{aligned}
$$

${ }^{44}(57)$ is the condition that expected payoffs are strictly concave in $\left(e_{i 1}\right) ;(58)$ is the condition that the Hessian of the expected payoff function has strictly positive determinant. 
With this notation, (58) can be written as

$$
K_{1}^{\prime \prime}\left(e_{i 1}\right) \cdot A+K_{2}^{\prime \prime}\left(e_{i 2}\right) \cdot B+C \leq K_{1}^{\prime \prime}\left(e_{i 1}\right) K_{2}^{\prime \prime}\left(e_{i 2}\right)
$$

To prove that (58) holds for sufficiently convex cost functions, suppose it does not hold for some pair of cost function $\widetilde{K}_{1}$ and $\widetilde{K}_{2}$. Let $\widehat{K}_{t}(e)=\widetilde{K}_{t}(e)+\frac{\kappa}{2} e^{2}$. Then (59) for $\widehat{K}_{1}$ and $\widehat{K}_{2}$ is

$$
\begin{gathered}
\widetilde{K}_{1}^{\prime \prime}\left(e_{i 1}\right) \cdot A+\widetilde{K}_{2}^{\prime \prime}\left(e_{i 2}\right) \cdot B+C \leq \\
\widetilde{K}_{1}^{\prime \prime}\left(e_{i 1}\right) \widetilde{K}_{2}^{\prime \prime}\left(e_{i 2}\right)+\kappa\left(\widetilde{K}_{1}^{\prime \prime}\left(e_{i 1}\right)+\widetilde{K}_{2}^{\prime \prime}\left(e_{i 2}\right)-A-B\right)+\kappa^{2}
\end{gathered}
$$

For all $A$ and $B$, the right-hand side of this inequality can be made arbitrarily high by increasing $\kappa$, so that the inequality is satisfied and thus (58) holds.

Local Second-Order Conditions In the symmetric equilibrium, $\Delta e_{i 1}=\Delta e_{i 2}=0$. Using this equation in (57) and (58), $f_{1}^{\prime}(0)=0$ and the symmetry of $f_{1}$ and $f_{2}$ (Assumption 2) gives

$$
\begin{gathered}
\eta^{2} W_{2} \int_{-\infty}^{\infty} f_{2}^{\prime}(\eta s) f_{1}(s) d s<K_{1}^{\prime \prime}\left(e_{i 1}\right) \\
\left(\frac{W_{2}}{K_{2}^{\prime \prime}\left(e_{i 2}\right)}+\frac{\eta^{2} W_{2}}{K_{1}^{\prime \prime}\left(e_{i 1}\right)}\right) \int_{-\infty}^{\infty} f_{2}^{\prime}(\eta s) f_{1}(s) d s \leq 1 .
\end{gathered}
$$

By Assumption 2, $f_{1}(s)=f_{1}(-s)$ and $f_{2}^{\prime}(\eta s)=-f_{2}^{\prime}(-\eta s)$. This implies that $\int_{-\infty}^{\infty} f_{2}^{\prime}(\eta s) f_{1}(s) d s=0$. Thus, the left-hand sides of (61) and (62) are all 0 and the inequalities hold automatically.

\subsubsection{Feedback Policy: Proof of Lemma $3^{45}$}

(10) and (18) imply

$$
\begin{gathered}
e_{2}^{*}\left(\eta, W-W_{1}, 0\right)-E\left(e_{2}^{*}\left(\eta, W-W_{1}, 1\right)\right)= \\
\left(K_{2}^{\prime}\right)^{-1}\left(\left(W-W_{1}\right) C(\eta)\right)-2 \int_{0}^{\infty}\left(K_{2}^{\prime}\right)^{-1}\left(f_{2}(\eta s)\left(W-W_{1}\right)\right) f_{1}(s) d s .
\end{gathered}
$$

\footnotetext{
${ }^{45}$ The proof resembles Aoyagi (2010) and Ederer (2010) (case with non-complementary abilities), but allows for $W_{1}>0$ and $\eta \neq 1$.
} 
Using Definition 3 and the symmetry of $f_{1}$ and $f_{2}$, the right-hand side can be written as

$$
\begin{aligned}
& \left(K_{2}^{\prime}\right)^{-1}\left(\left(W-W_{1}\right) \int_{-\infty}^{\infty} f_{2}(\eta s) f_{1}(s) d s\right) \\
& -\int_{\infty}^{\infty}\left(K_{2}^{\prime}\right)^{-1}\left(f_{2}(\eta s)\left(W-W_{1}\right)\right) f_{1}(s) d s .
\end{aligned}
$$

Substituting $g(s) \equiv\left(W-W_{1}\right) f_{2}(\eta s)$, this becomes

$$
\left(K_{2}^{\prime}\right)^{-1}\left(\int_{-\infty}^{\infty} g(s) f_{1}(s) d s\right)-\int_{-\infty}^{\infty}\left(K_{2}^{\prime}\right)^{-1}(g(s)) f_{1}(s) d s .
$$

According to Jensen's inequality, this expression is weakly negative (weakly positive) if $\left(K_{2}^{\prime}\right)^{-1}$ is convex (concave), which is the case if and only if $K_{2}^{\prime}$ is concave (convex), that is, $K_{2}^{\prime \prime \prime} \leq 0\left(K_{2}^{\prime \prime \prime} \geq 0\right)$.

\subsubsection{Optimal prizes with general cost functions}

For the no feedback case, we again restrict the third derivative of the cost functions in such a way that the feedback policy is optimal by Corollary 6 .

Lemma 8 Suppose $K_{t}^{\prime \prime \prime} \geq 0$ for $t=1,2$. For all $\eta>0$

(i) $W_{1}^{P}(\eta, 0)=0$ if

$$
\frac{f_{1}(0)-\eta C(\eta)}{K_{1}^{\prime \prime}\left[\left(K_{1}^{\prime}\right)^{-1}(\eta W C(\eta))\right]}-\frac{C(\eta)}{K_{2}^{\prime \prime}\left[\left(K_{2}^{\prime}\right)^{-1}(W C(\eta))\right]}<0 .
$$

(ii) $W_{1}^{P}(\eta, 0)=W$ if

$$
\frac{f_{1}(0)-\eta C(\eta)}{K_{1}^{\prime \prime}\left[\left(K_{1}^{\prime}\right)^{-1}\left(f_{1}(0) W\right)\right]}-\frac{C(\eta)}{K_{2}^{\prime \prime}(0)}>0 .
$$

(iii) If neither (63) nor (64) holds, $W_{1}^{P} \in[0, W]$.

Proof. Using (17) and (18) in (13) gives

$$
\begin{gathered}
V^{P}\left(\eta, W_{1}, 0\right)= \\
\left(K_{1}^{\prime}\right)^{-1}\left(f_{1}(0) W_{1}+\eta\left(W-W_{1}\right) C(\eta)\right)+\left(K_{2}^{\prime}\right)^{-1}\left(\left(W-W_{1}\right) C(\eta)\right) .
\end{gathered}
$$


This yields

$$
\begin{gathered}
\frac{\partial V^{P}\left(\eta, W_{1}, 0\right)}{\partial W_{1}}= \\
\frac{f_{1}(0)-\eta C(\eta)}{K_{1}^{\prime \prime}\left[\left(K_{1}^{\prime}\right)^{-1}\left(f_{1}(0) W_{1}+\eta\left(W-W_{1}\right) C(\eta)\right)\right]}-\frac{C(\eta)}{K_{2}^{\prime \prime}\left[\left(K_{2}^{\prime}\right)^{-1}\left(\left(W-W_{1}\right) C(\eta)\right)\right]}
\end{gathered}
$$

and

$$
\begin{gathered}
\frac{\partial^{2} V^{P}\left(\eta, W_{1}, 0\right)}{\partial W_{1}^{2}}= \\
-\frac{\left(f_{1}(0)-\eta C(\eta)\right)^{2} \cdot K_{1}^{\prime \prime \prime}\left[\left(K_{1}^{\prime}\right)^{-1}\left(f_{1}(0) W_{1}+\eta\left(W-W_{1}\right) C(\eta)\right)\right]}{\left(K_{1}^{\prime \prime}\left[\left(K_{1}^{\prime}\right)^{-1}\left(f_{1}(0) W_{1}+\eta\left(W-W_{1}\right) C(\eta)\right)\right]\right)^{3}} \\
-\frac{(C(\eta))^{2} \cdot K_{2}^{\prime \prime \prime}\left[\left(K_{2}^{\prime}\right)^{-1}\left(\left(W-W_{1}\right) C(\eta)\right)\right]}{\left(K_{2}^{\prime \prime}\left[\left(K_{2}^{\prime}\right)^{-1}\left(\left(W-W_{1}\right) C(\eta)\right)\right]\right)^{3}}
\end{gathered}
$$

Since $K_{t}^{\prime \prime}>0, K_{t}^{\prime \prime \prime} \geq 0$ implies $\frac{\partial^{2} V^{P}\left(\eta, W_{1}, 0\right)}{\left(\partial W_{1}\right)^{2}} \leq 0$.

(i) Thus, the principal will set $W_{1}=0$ provided

$$
\left.\frac{\partial V^{P}\left(\eta, W_{1}, 0\right)}{\partial W_{1}}\right|_{W_{1}=0}=\frac{f_{1}(0)-\eta C(\eta)}{K_{1}^{\prime \prime}\left[\left(K_{1}^{\prime}\right)^{-1}(\eta W C(\eta))\right]}-\frac{C(\eta)}{K_{2}^{\prime \prime}\left[\left(K_{2}^{\prime}\right)^{-1}(W C(\eta))\right]}<0 .
$$

(ii) She will set $W_{1}=W$ provided

$$
\left.\frac{\partial V^{P}\left(\eta, W_{1}, 0\right)}{\partial W_{1}}\right|_{W_{1}=W}=\frac{f_{1}(0)-\eta C(\eta)}{K_{1}^{\prime \prime}\left[\left(K_{1}^{\prime}\right)^{-1}\left(f_{1}(0) W\right)\right]}-\frac{C(\eta)}{K_{2}^{\prime \prime}(0)}>0
$$

incentives relative to the unconstrained case. To make up for this, she has to adjust the weight of first-period performance downwards. 